\title{
Coherent acoustic phonons in van der Waals nanolayers and heterostructures
}

\author{
J. D. G. Greener, ${ }^{1}$ A. V. Akimov, ${ }^{1,}{ }^{*}$ V. E. Gusev,${ }^{2}$ Z. R. Kudrynskyi, ${ }^{1}$ P. H. Beton, ${ }^{1}$ Z. D. Kovalyuk,,${ }^{3}$ T. Taniguchi, ${ }^{4}$ \\ K. Watanabe, ${ }^{4}$ A. J. Kent, ${ }^{1}$ and A. Patanè ${ }^{1}$ \\ ${ }^{1}$ School of Physics and Astronomy, The University of Nottingham, Nottingham NG7 2RD, United Kingdom \\ ${ }^{2}$ LAUM, UMR-CNRS 6613, Le Mans Université, Avenue O. Messiaen, 72085 Le Mans, France \\ ${ }^{3}$ Institute for Problems of Materials Science, The National Academy of Sciences of Ukraine, Chernivtsi, Ukraine \\ ${ }^{4}$ National Institute for Materials Science, 1-1 Namiki, Tsukuba, Ibaraki 305-0044, Japan
}

(Received 22 March 2018; revised manuscript received 31 May 2018; published 9 August 2018)

\begin{abstract}
Terahertz (THz) and sub-THz coherent acoustic phonons have been successfully used as probes of various quantum systems. Since their wavelength is in the nanometer range, they can probe nanostructures buried below a surface with nanometer resolution and enable control of electrical and optical properties on a picosecond time scale. However, coherent acoustic phonons have not yet been widely used to study van der Waals (vdW) two-dimensional (2D) materials and heterostructures. This class of 2D systems features strong covalent bonding of atoms in the layer planes and weak van der Waals attraction between the layers. The dynamical properties of the interface between the layers or between a layer and its supporting substrate are often omitted as they are difficult to probe. On the other hand, these play a crucial role in interpreting experiments and/or designing new device structures. Here, we use picosecond ultrasonic techniques to investigate phonon transport in vdW InSe nanolayers and InSe/hBN heterostructures. Coherent acoustic phonons are generated and detected in these 2D systems and allow us to probe elastic parameters of different layers and their interfaces. In particular, our study of the elastic properties of the interface between vdW layers reveals a strong coupling over a wide range of frequencies up to $0.1 \mathrm{THz}$, offering prospects for high-frequency electronics and technologies that require control over the charge and phonon transport across an interface. In contrast, we reveal a weak coupling between the InSe nanolayers and sapphire substrates, relevant in thermoelectrics and sensing applications, which can require quasi-suspended layers.
\end{abstract}

DOI: 10.1103/PhysRevB.98.075408

\section{INTRODUCTION}

During the last decade, techniques for the generation and detection of coherent acoustic phonons with frequencies from tens of gigahertz $(\mathrm{GHz})$ to several terahertz $(\mathrm{THz})$ have enabled novel approaches to the investigation of a wide range of materials and nanostructures. Experiments with coherent phonons were made possible by the availability of lasers for the generation of ultrashort light pulses. This has led to a new field of research, picosecond ( $p s$ ) ultrasonics, which has extended traditional acoustics and ultrasonics to higher frequencies and shorter wavelengths $[1,2]$, thus stimulating and advancing topical research areas in condensed matter physics, including the following.

(i) Phonon transport. Of particular interest is phonon transport through the interface between two nanostructures or between one system and its environment [3-9]. Acoustic mismatch theory, which describes phonon transport through an elastically perfect interface with strong bonds, does not

\footnotetext{
*Corresponding author: andrey.akimov@nottingham.ac.uk
}

Published by the American Physical Society under the terms of the Creative Commons Attribution 4.0 International license. Further distribution of this work must maintain attribution to the author(s) and the published article's title, journal citation, and DOI. always apply. Thus different approaches are often required to understand and control phonon transport in real systems.

(ii) Phonon interactions. Interactions of coherent phonons with thermal phonons [10], electrons [11-15], plasmons [16-18], and magnons [19-25] are intensively studied in metallic (for a review see Ref. [26]) and semiconductor [27,28] nanoparticles, multilayered heterostructures [13-15,29,30], and patterned surfaces [16-18,21,31]. These interactions underpin a wide range of phenomena, such as piezoelectricity, light scattering, phonon-assisted tunneling, etc., and the design and successful development of devices for electronics [32] and optoelectronics [33].

(iii) Phonon nanoscopy. Coherent phonons with $\mathrm{THz}$ frequency can probe interfaces and nanoobjects buried below a surface with atomic depth resolution (e.g., $\ll 1 \mathrm{~nm}$ ). Since its first use to probe and control the quality of microchip contacts [34], nanoscopy has been used to image interfaces [35,36], chemical reactions [37], and biological cells [38]. Also, acoustic, optic and acousto-optic parameters of inhomogeneous materials can be probed with a nanometer spatial depth resolution [39].

Of critical importance in the studies highlighted in (i)(iii) is the generation and detection of quantized coherent phonons in various nanoobjects (e.g., in single nanoparticles $[9,26,27,40,41]$ and quantum wires [42]). In this case, the phonon spectrum consists of well-defined resonances each with a linewidth $\Delta f$ that is smaller than the resonance 
frequency $f$, and finesse defined by the quality factor $Q=$ $f / \Delta f$. A number of recent works have focused on twodimensional (2D) nanometer-thick layers where phonons are quantized in the direction perpendicular to the $2 \mathrm{D}$ plane. Examples include submicrometer free-standing membranes based on Si [43], GaAs [44] and GaN [45], and phonon nanocavities [46].

Among 2D systems, van der Waals (vdW) crystals have emerged as a new class of materials for several potential applications. In a vdW crystal, the atoms in each layer are bound by strong covalent bonds, whereas the planes are held together by weak vdW interactions. Thus several physical properties of these materials, including elastic properties (e.g., sound velocity) are strongly anisotropic. The extended family of $\mathrm{vdW}$ crystals includes graphene, transition metal dichalchogenides (e.g., $\mathrm{MoS}_{2}, \mathrm{WS}_{2}$, etc), metal chalcogenides (e.g., InSe, GaSe, etc.), hexagonal boron nitride ( $\mathrm{hBN}$ ), and many others $[47,48]$. For this new class of materials, picosecond acoustics could help to address several important open questions. The interface between two vdW layers or between a vdW layer and its supporting substrate may or may not be elastically perfect, thus influencing thermal and charge transport. For example, the control of phonon transport across a vdW interface could provide a strategy to probe and tune thermal conductivity for specific applications in electronics and thermoelectrics [47-51]. The interaction of coherent phonons with charge carriers could enable new routes for the generation of microwave frequencies as it is done in nanoelectromechanical (NEMS) systems in the $\mathrm{MHz}-\mathrm{GHz}$ frequency range [52], in piezoelectric heterostructures for the sub-THz range [53], and traditional semiconductor devices such as Schottky diodes $[11,54]$ and tunneling devices [12]. Also, due to strong elastic anisotropy and phonon quantization, $2 \mathrm{D} \mathrm{vdW}$ layers could provide an ideal system for phonon nanoscopy and nondestructive sensitive imaging of molecules and cells coupled to the nanolayers by vdW forces. Although these research areas are still in their infancy, recent experimental works have demonstrated the possibility of generating coherent phonons in vdW nanolayers, including graphene [6], $\mathrm{WSe}_{2}$ [55], $\mathrm{WTe}_{2}$ [56], $\mathrm{MoS}_{2}$ [57], and InSe [58].

Here, we use coherent phonons with frequencies from 10 to $100 \mathrm{GHz}$, generated and detected using ultrafast optical (pump-probe) methods, for the nondestructive investigation of nanomechanical oscillations in InSe nanolayers and InSe/hBN heterostructures. These studies enable us to probe the $\mathrm{vdW}$ bonding between the layers and their adhesion to the substrate. The $\mathrm{GHz}$ and sub-THz nanomechanical oscillations have frequency, amplitude, and spectral width that depend on the stiffness of the interfacial bonds at the interface. In particular, we find that the interface between InSe and hBN can be described by acoustic mismatch over a wide frequency range $f \leqslant 100 \mathrm{GHz}$.

The paper is organized as follows. Section II describes our samples and the experimental techniques used for the generation and detection of coherent phonons. Section III introduces the theoretical background used to describe the resonant phonon modes in vdW nanolayers and heterostructures. Section IV presents the measured and calculated phonon spectra for a variety of samples based on InSe nanolayers and $\mathrm{InSe} / \mathrm{hBN}$ heterostructures. The main findings are discussed in Sec. V taking into account the properties of interfaces and phonon scattering processes. Conclusions and outlook for future studies and applications are presented in Sec. VI.

\section{EXPERIMENTAL TECHNIQUES}

\section{A. VdW InSe nanolayers and heterostructures}

Our experiments focus on InSe vdW crystals and InSe/hBN heterostructures. The InSe represents a relatively new addition to the family of $2 \mathrm{D} \mathrm{vdW}$ crystals and is receiving an increasing interest due to its unique and versatile electronic properties. This compound has a band gap that increases markedly with decreasing layer thickness down to a single layer offering full coverage of the photonic spectrum from the violet to the infrared range [59-61]; it has relatively light $\left(m_{c} \sim 0.14 m_{e}\right)$ conduction band electrons with high electron mobility $(\mu)$ even in the limit of atomically thin films $\left(\mu=0.2 \mathrm{~m}^{2} / \mathrm{Vs}\right.$ at $300 \mathrm{~K}$ ) [60]; furthermore, the encapsulation of InSe by hBN has enabled the fabrication of high-quality field effect transistors (FETs) where the hBN capping layer isolates the InSe from the environment and also serves as dielectric layer for electrostatic gating [60]. Phonon transport and thermal conduction in 2D InSe and its heterostructures are still largely unexplored. To date, theoretical studies have focussed on phonon properties and in-plane transport of the longitudinal, transverse, and flexural acoustic modes in single layers [62,63]. Experimental work has largely been limited to Raman studies $[59,61]$ and elastic properties (e.g., sound velocity and elastic constants) are only known for bulk InSe [64].

Our 2D InSe layers were prepared by exfoliation of Bridgman-grown crystals of rhombohedral $\gamma$-InSe [59]. They have areas of about $10-100 \mu \mathrm{m}^{2}$ and thicknesses from $\sim 5$ to $\sim 100 \mathrm{~nm}$. After the exfoliation, the individual InSe flakes were laid onto another layered crystal (e.g., InSe or hBN) so that the adhesive $\mathrm{vdW}$ force between the layers forms a homojunction (e.g., InSe on InSe) or a heterojunction (e.g., InSe on $\mathrm{hBN}$ or hBN on InSe). These samples were transferred on a $c$-cut sapphire $\left(\mathrm{Al}_{2} \mathrm{O}_{3}\right)$ substrate with an atomically flat surface (surface roughness of $\sim 0.1 \mathrm{~nm}$ ). The thickness and the surface roughness of the layers were assessed by atomic force microscopy (AFM) in noncontact mode under ambient conditions. Representative AFM data and their discussion are presented in Sec. V.

Figure 1(a) shows the optical images for an InSe/hBN heterostructure and the individual InSe and hBN layers prior to stamping of hBN on InSe with thickness $a=(81 \pm 1) \mathrm{nm}$ and $b=(59 \pm 1) \mathrm{nm}$, respectively. Room temperature $(T=$ $300 \mathrm{~K}) \mu$-photoluminescence ( $\mu \mathrm{PL}$ ) and $\mu$-Raman maps, and representative spectra are shown in Figs. 1(b)-1(e). By comparing the spectra acquired in areas with and without the hBN layer, we find that the encapsulation of InSe by hBN does not modify the energy of the Raman modes and/or of the PL emission of pristine InSe. However, it induces a significant increase of both Raman and PL signals. This behavior, which is not observed when the hBN layer lies below the InSe layer, is indicative of an increased absorption of light by the InSe layer due a smaller reflection of the laser light at the interface between air and hBN (refractive index $n_{\mathrm{hBN}}=1.8$ [65]), compared to air $\left(n_{\text {air }}=1\right)$ and InSe $\left(n_{\text {InSe }}=2.7\right.$ [66]). 

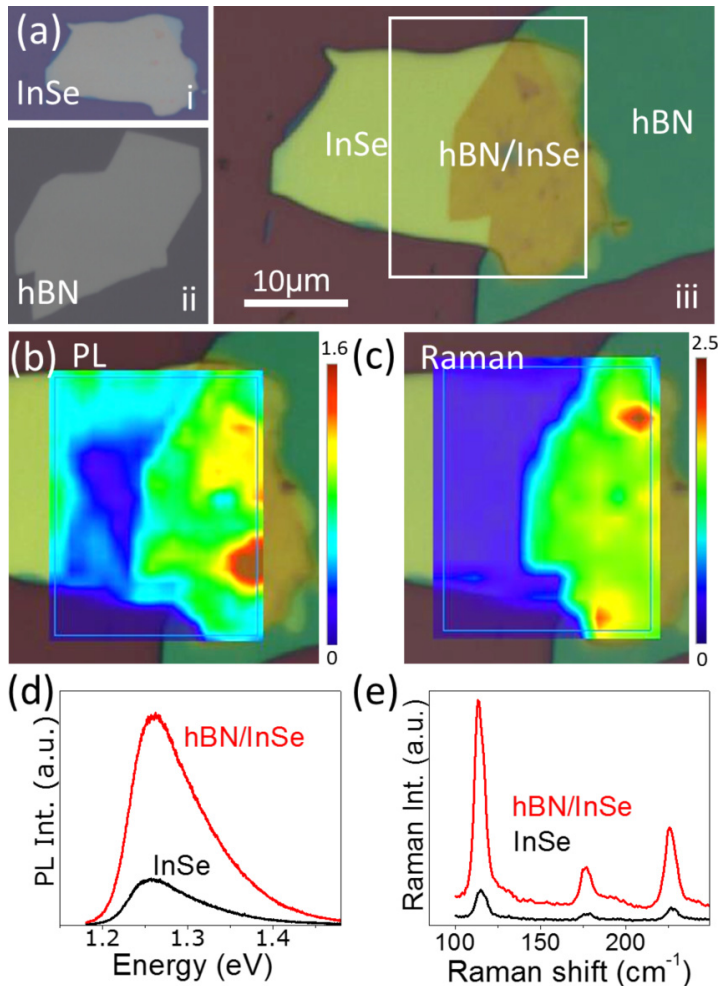

(e)

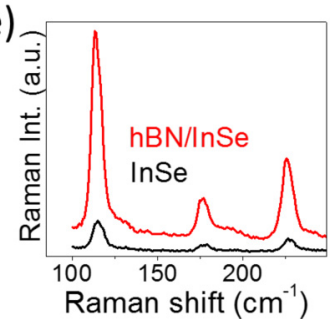

FIG. 1. (a) Optical images of InSe and hBN layers before [(i) and (ii)] and after (iii) their stacking to form an InSe/hBN heterostructure on a $\mathrm{SiO}_{2}$ substrate. The rectangle in part iii is mapped by $\mu \mathrm{PL}$ and $\mu$ Raman in part [(b) and (c)]. [(b) and (c)] Maps of the $\mu$ PL (b) and $\mu$ Raman (c) intensities for the sample shown in part (a-iii). Each map is overlaid on the optical image of the sample and shows the InSe and hBN/InSe areas. [(d) and (e)] Representative $\mu \mathrm{PL}$ (d) and $\mu$ Raman (e) spectra for InSe before (black) and after (red) the stamping of the hBN layer.

\section{B. Generation and detection of coherent acoustic phonons}

We use femtosecond lasers and the most common pumpprobe picosecond acoustics method to generate and detect coherent phonons. This method, first proposed by Thomson et al. [67] in the 1980s, is well developed and is described in several reviews [1,2]. In this method, an optical pulse from a pump femtosecond laser excites the layers directly or through an optoelastic transducer (e.g., a thin metal film), which absorbs light. As a result, a stress is generated in the sample due to a rapid, almost instantaneous electron and lattice temperature rise [67-69]. The stress causes dynamical strain and, correspondingly, generates a coherent wave packet of longitudinal acoustic phonons. The spectrum of these sub-THz phonons depends on the absorption length and the thickness of the layer. The spatial and temporal evolutions of the coherent phonons are governed by the elastic properties of the materials and their interfaces and can be probed by a second optical pulse from the same pump laser or from another probe laser synchronized with the pump one. Scanning the time delay between pump and probe pulses makes it possible to monitor the temporal evolution of the wave packet of coherent acoustic phonons generated in the sample.

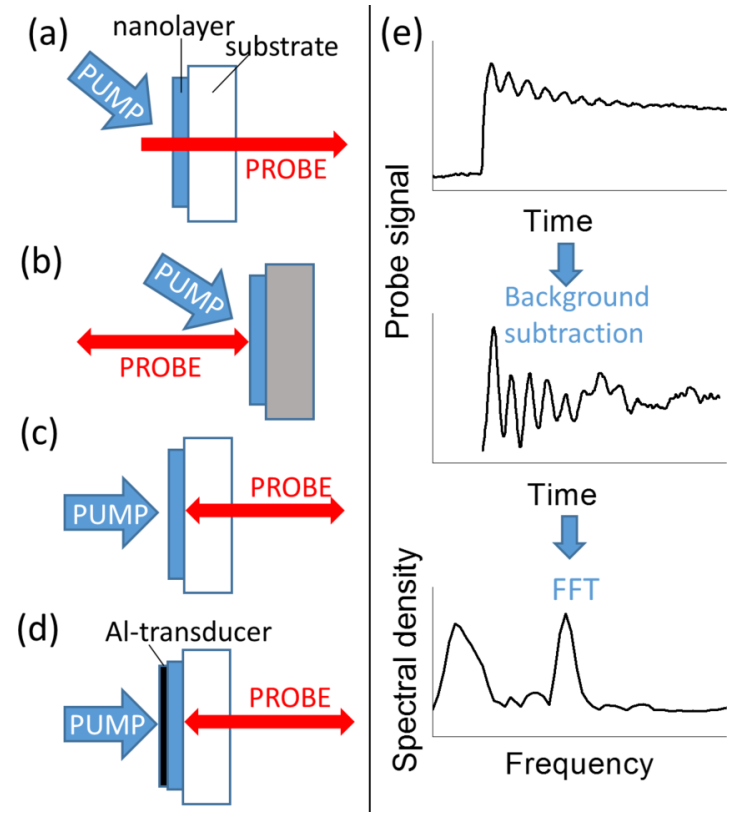

FIG. 2. (a)-(d) Schemes for pump-probe experiments in transmission (a) and reflection [(b), (c), and (d)] geometries. The stress in the nanolayers is excited directly [(a)-(c)] or through an Al-transducer (d). (e) A generic pump-probe signal and its analysis to deduce the phonon spectrum: (top) temporal signal; (middle) temporal signal after subtraction of a slow decaying background; and (bottom) phonon spectrum obtained by fast Fourier transform (FFT) of the temporal signal after subtraction of the background. In this example, the FFT spectrum shows two phonon resonances.

For our studies, we use two 120-fs pulsed Ti:sapphire lasers with wavelength $\lambda \approx 800 \mathrm{~nm}$ and repetition rate of 80 MHz. The experiments were carried out using an asynchronous optical sampling (ASOPS) technique [70] and also the traditional method in which the laser output is split into pump and probe beams. The pump beam passes through an acoustooptic modulator and frequency doubler. The pump pulse with wavelength $\lambda \approx 400 \mathrm{~nm}$ is focused onto the sample to an area of $\sim 20 \times 20 \mu \mathrm{m}^{2}$ for normal incidence and $\sim 20 \times 30 \mu \mathrm{m}^{2}$ for $45^{\circ}$ incidence. The probe beam with $\lambda \approx 800 \mathrm{~nm}$ is focused on the sample surface to a diameter of several microns, overlapping spatially with the pump spot. The power for pump and probe were kept below 20 and $0.5 \mathrm{~mW}$, respectively, to avoid nonlinear effects and photooxidation of the InSe layers [71]. We used four experimental schemes shown in Figs. 2(a)-2(d) in which the intensities of the transmitted [Fig. 2(a)] or reflected [Figs. 2(b)-2(d)] probe beams are measured. The pump beam is directed at an angle of about $45^{\circ}$ [Fig. 2(a) and 2(b)] or is collinear and in the opposite direction to the incident probe beam [Figs. 2(c) and 2(d)]. For coherent phonon generation in thin $(<70 \mathrm{~nm})$ layers, we used Al transducers [Fig. 2(d)]. The Al films were deposited on the vdW layers by electron beam evaporation and their thicknesses $(\sim 30 \mathrm{~nm})$ were measured by AFM.

Figure 2(e) demonstrates the procedure for obtaining the spectrum of coherent phonons from the temporal evolution of an arbitrary pump-probe signal. The top panel shows schematically a typical pump-probe temporal trace, which 
includes an instantaneous rise and a slow decay modulated by periodic oscillations. In a picosecond acoustics experiment, these oscillations arise from coherent acoustic phonons and can be revealed more clearly by subtracting to the signal a slow decay background, as fitted by a polynomial or exponential function [see the middle panel in Fig. 2(e)]. The spectrum of coherent phonons is then obtained by performing a fast Fourier transform (FFT) of the subtracted curve [bottom panel of Fig. 2(e)]. The procedure of subtracting a slow-decaying background may cause artifacts showing low-frequency lines with $Q \sim 1$ in the FFT spectrum. Thus the frequency range from 0 to $10 \mathrm{GHz}$ is not considered in the analysis of the FFT spectra. The relative intensities of the lines in the phonon spectrum are generally difficult to model as they are determined by photoelastic parameters [72], which are not known for the studied materials. On the other hand, we can analyze the frequencies and linewidths of the phonon resonances. These are governed by the main elastic properties of the layers and can be compared with the values obtained by solving elastic equations (see Sec. III and Appendix).

\section{THEORETICAL BACKGROUND}

We describe coherent phonons as classical wave packets of acoustic waves with displacement vector $\mathbf{u}$, wavevector $\mathbf{k}$ and given strain, $\varepsilon$, and stress, $\sigma$, tensors. For propagation of longitudinal acoustic phonons $(\mathbf{u} \| \mathbf{k})$ along the $x$ direction, perpendicular to the plane of the vdW layers, the solution of the elastic equations for the Fourier component $\omega$ in the $i$ th layer is

$$
\tilde{u}(\omega, x)=A_{i} \cos \left(k_{i} x\right)+B_{i} \sin \left(k_{i} x\right),
$$

where $k_{i}=\omega / s_{i}$ is the phonon wavevector and $s_{i}$ is the sound velocity in the $i$ th layer (for details see Appendix). The solution for the elastic wave in a semi-infinitive substrate is

$$
\tilde{u}_{S}(\omega, x)=A_{S} \exp \left(-i k_{i} x\right) .
$$

The frequency dependent coefficients $A_{i}, B_{i}$, and $A_{S}$ define the coherent phonon spectrum and can be found by applying boundary conditions to the displacement and stress at each interface. The simplest, most common case for the boundary conditions is the acoustic mismatch (AM) model, i.e., the displacement and stress are continuous at each interface [73]. This approximation works for relatively thick layers and perfect, atomically flat interfaces. An example of AM is shown schematically in Fig. 3(a) for an InSe/hBN heterostructure on a substrate. Here, the elastic coupling between the boundary atoms in the two materials is assumed to be absolutely rigid. For the wave propagating in a medium $(i)$ and reaching the interface with another medium $(j)$, the amplitude reflection coefficient $R_{i j}$ for the strain is

$$
R_{i j}=\frac{Z_{j}-Z_{i}}{Z_{j}+Z_{i}}
$$

where $Z_{i}$ and $Z_{j}$ are the acoustic impedances $(Z=\rho s$, where $\rho$ is the density and $s$ is the sound velocity) of the materials. For phonons propagating from a high to a low impedance material $\left(Z_{i}>Z_{j}\right), R_{i j}<0$ and the phase for the strain wave of the reflected beam is changed by $\pi$. In the opposite case $\left(Z_{i}<Z_{j}\right)$, (a)

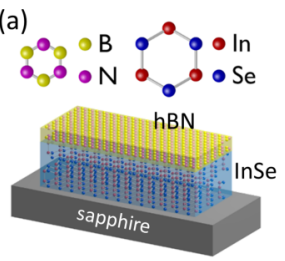

(b)

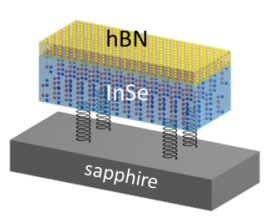

(c)

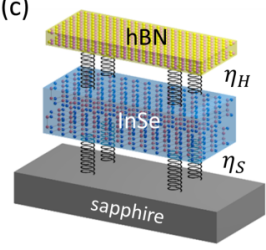

FIG. 3. Schematics showing different strengths of elastic coupling between van der Waals layers (e.g., InSe and hBN) and between a van der Waals layer and a sapphire substrate. (a) Rigid interface between InSe and hBN, and between InSe and sapphire. The elastic properties of the rigid interfaces are described by the acoustic mismatch model. (b) Rigid interface between InSe and hBN, but weak elastic coupling between InSe and sapphire. (c) Weak elastic coupling for both InSe/hBN and InSe/sapphire interfaces, as described by springs with stiffnesses $\eta_{H}$ and $\eta_{S}$, respectively.

$R_{i j}>0$ and the phase does not change on reflection. The case $Z_{i}=Z_{j}$ corresponds to $R_{i j}=0$.

When $Z_{i} \neq Z_{j}$, the interference of waves reflected at an interface results in phonon quantization and nanomechanical resonances. For a single layer of thickness $a$ on a substrate with acoustic impedance $Z_{S}$, the phonon frequencies are given by

$$
f_{n}=\frac{(2 n-1) s}{4 a}, \text { for } Z_{i}<Z_{S}
$$

and

$$
f_{n}=\frac{n s}{2 a}, \text { for } Z_{i}>Z_{S}
$$

where $n$ is an integer number.

In general, the AM model may not describe well the phonon transport in thin layers. Also, it may not be valid when the phonon dispersion plays a role, e.g., at high phonon frequencies or when the interface between the layers is not elastically perfect. Here we consider layers with thicknesses $a>5 \mathrm{~nm}$, larger than the vdW interlayer separation, i.e., $\sim 0.8 \mathrm{~nm}$ and $0.7 \mathrm{~nm}$ in InSe and hBN, respectively (see Table I). Also, the maximum phonon frequency studied in this work is $120 \mathrm{GHz}$, which is much smaller than the frequency at which phonon dispersion effects become important $(\sim 1 \mathrm{THz})$. Thus the main limitation to the validity of the AM model to our structures is

TABLE I. Elastic parameters of InSe, hBN, and Al used in the calculations.

\begin{tabular}{lccrr}
\hline \hline & InSe & hBN & Al & Sapphire \\
\hline Density, $\mathrm{kg} / \mathrm{m}^{3}$ & 5500 & 2180 & 2700 & 4000 \\
LA sound velocity, m/s & $2500^{\mathrm{a}}$ & $3440^{\mathrm{b}}$ & 6420 & 11000 \\
Acoustic impedance, MPa s/m & 14 & 7.5 & 17 & 44 \\
Elastic constant, $\mathrm{C}_{33}, \mathrm{GPa}$ & 36 & 26 & & \\
vdW interlayer period, nm & 0.8 & 0.7 & & \\
Spring stiffness of vdW bond, & 3.7 & 3.4 & & \\
$10^{19} \mathrm{~N} / \mathrm{m}^{3}$ & & & & \\
\hline
\end{tabular}

\footnotetext{
${ }^{\mathrm{a}}$ Reference [64].
}

${ }^{\mathrm{b}}$ Reference [75]. 
the imperfectness of the interfaces. To account for the elastic properties of an imperfect interface, we use the spring model [5,74]: the adjacent layers of two materials, $i$ and $j$, are coupled by effective "springs" with stiffness $\eta_{i j}$. Figure 3(b) shows the scenario in which an InSe/hBN heterostructure on sapphire has AM between the layers, but is weakly coupled to the substrate by an effective spring of stiffnesses $\eta_{S}$. Figure 3(c) shows the scenario in which both interfaces are imperfect, i.e. there is also a spring of stiffness $\eta_{H}$ at the InSe/hBN interface.

The boundary conditions in the spring model include the continuity of the stress at the interfaces with springs, from which the coefficients $A_{i}, B_{i}$, and $A_{S}$ can be derived (see Appendix). Similarly to the AM model, the spring model reveals nanomechanical resonances in the heterostructure. However, the resonance frequencies $f_{n}$ differ from those predicted by the AM model; also, due to the weaker coupling at the interfaces, these resonances can be narrower.

For acoustic phonons propagating from material $i$ to material $j$ and back from $j$ to $i$, it is convenient to use parameters $G_{i j}$ and $G_{j i}$, respectively, related to $\eta_{i j}=\eta_{j i}$ :

$$
G_{i j}=G_{j i}=\frac{\eta_{i j}\left(Z_{i}+Z_{j}\right)}{2 \pi Z_{i} Z_{j}} .
$$

Since in our work we use no more than two vdW nanolayers, we consider two parameters $G_{H}$ and $G_{S}$, which characterize the coupling between the vdW nanolayers and between the inner nanolayer with the substrate, respectively,

$$
G_{H}=\frac{\eta_{H}\left(Z_{1}+Z_{2}\right)}{2 \pi Z_{1} Z_{2}}
$$

and

$$
G_{S}=\frac{\eta_{S}\left(Z_{S}+Z_{2}\right)}{2 \pi Z_{S} Z_{2}},
$$

where $Z_{1}, Z_{2}$, and $Z_{S}$ are the acoustic impedances of the outer, inner nanolayers, and substrate, respectively. The parameters $G_{H}$ and $G_{S}$ have the dimension of frequency. For a single layer weakly coupled to the substrate, e.g., $G_{S} \ll f_{n}$, the resonance frequencies are given by Eq. (5).

\section{MEASURED AND MODELLED PHONON SPECTRA}

This section presents the main experimental findings. The first section, Sec. IV A, focuses on nanomechanical resonances in InSe nanolayers and explores their dependence on the InSe layer thickness and elastic coupling with the substrate. Section IV B concentrates on vdW heterostructures and demonstrates that the AM model is well suited to describe a vdW interface, indicating an elastically ideal interface and strong interlayer coupling for both homo (e.g., InSe/InSe) and hetero (e.g., InSe/hBN) interfaces over a wide range of sub- $\mathrm{THz}$ frequencies.

One of the main aims of the present work is to obtain the values of $G_{S}$ or $G_{H}$, which are governed by the coupling parameters $\eta_{S}$ and $\eta_{H}$ for single nanolayers and heterostructures, respectively. To obtain these parameters, first, we obtain the frequencies $f_{n}$ of the phonon resonances from the measured phonon spectra and compare them with the theoretical values of $f_{n}$ obtained from the calculated spectra for various $G_{S}\left(G_{H}\right)$.

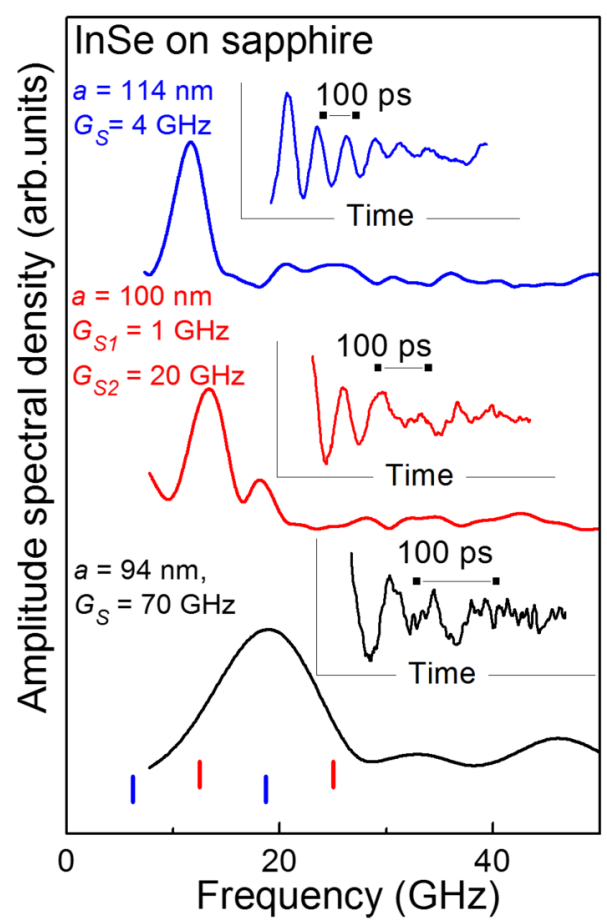

FIG. 4. Phonon spectra for different InSe nanolayers on a sapphire substrate. The insets show the temporal evolution of the pumpprobe signals after background subtraction. Vertical bars indicate the calculated phonon frequencies for an InSe nanolayer (thickness $a=100 \mathrm{~nm}$ ) coupled to the sapphire substrate in the limit of strong (blue bars, $G_{S} \gg f_{n}$ ) and weak (red bars, $G_{S} \ll f_{n}$ ) elastic coupling. The values of $G_{S}$ are deduced from the best fits of the spring model to the data.

To make this comparison, we calculate the modeled strain spectrum as

$$
S(f) \sim f \sqrt{\sum_{i}\left(\left|A_{i}\right|^{2}+\left|B_{i}\right|^{2}\right)},
$$

with the bulk elastic parameters from Table I and the layer thicknesses, as measured by AFM. We find that the coupling parameters $G_{S}\left(G_{H}\right)$ do not depend directly on the thickness of the nanolayers and are governed by the roughness of the surface at the interface with coupling parameter $\eta_{S}\left(\eta_{H}\right)$.

\section{A. InSe nanolayers}

Experiments on relatively thick $(a \sim 100 \mathrm{~nm})$ InSe layers were carried out using the scheme shown in Fig. 2(a). Figure 4 shows the measured coherent phonon spectra for three separate InSe layers with approximately the same thickness $a \sim 100 \mathrm{~nm}$ on a sapphire substrate. The background free temporal evolutions are also shown in the insets of Fig. 4. It can be seen that all spectra consist of a distinct spectral line between 10 and $20 \mathrm{GHz}$. Despite the layers having similar thickness, the lines are centered at different frequencies and the spectral width is twice larger for the lowest curve. Blue and red vertical bars indicate the phonon frequencies calculated from Eqs. (4) and (5), which correspond to AM and weak elastic coupling of the layers with the substrate $\left(G_{S} \ll f\right)$, respectively. The values of $G_{S}$ obtained from the comparison of the measured and 


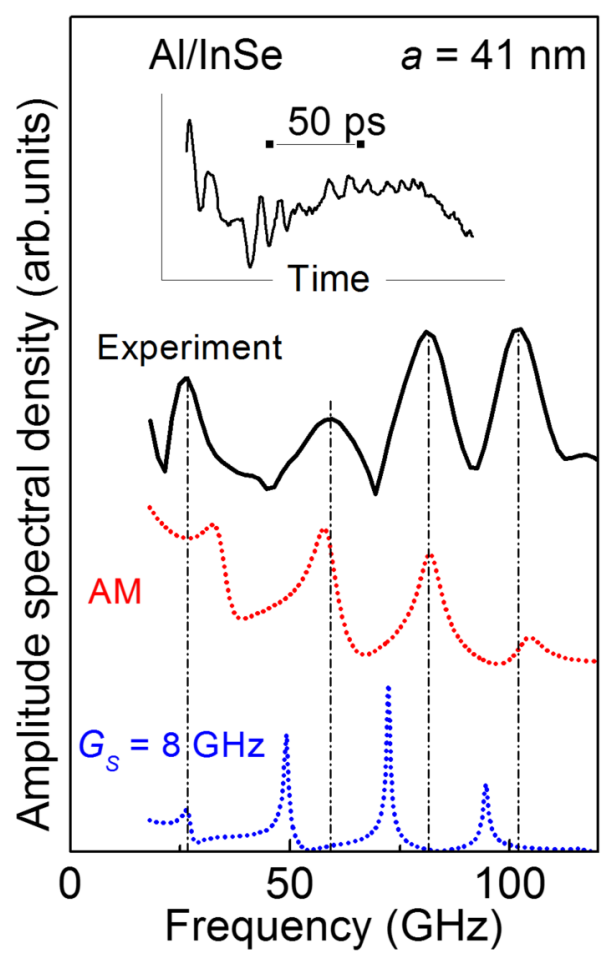

FIG. 5. Measured (solid lines) and calculated (dotted lines) phonon spectra for InSe nanolayers with thicknesses $a=41 \mathrm{~nm}$ on a sapphire substrate. Red and blue curves correspond to acoustic matching and weak coupling of the InSe nanolayer to the sapphire substrate, respectively. Phonons are excited via a $33-\mathrm{nm}$-thick Al transducer. Vertical dashed-dotted lines indicate the central frequencies of the measured phonon resonances. The inset shows the background free temporal evolution of the measured signal.

calculated spectra are shown in the same graph. The resonance at the top of Fig. 4 indicates a regime of weak coupling of InSe to the substrate; the one at the bottom has a broad spectral line centered at a frequency equal to that expected from AM; the middle spectrum shows instead two lines that cannot be described by a single coupling parameter $G_{S}$ : the spectral lines centered at 13.4 and $18.0 \mathrm{GHz}$ are reproduced by the spring model with $G_{S}=1$ and $20 \mathrm{GHz}$, respectively. Thus, from this study, we conclude that InSe layers on sapphire can exhibit different mechanical properties and elastic coupling to the substrate.

Experiments with thinner InSe layers were performed using the scheme shown in Fig. 2(d) using an $\mathrm{Al}$ transducer. The deposition of the $\mathrm{Al}$ film by electron beam evaporation on InSe produced uniform high-quality thin $(33 \mathrm{~nm})$ film. Thus we expect strong elastic coupling with $G_{i j} R_{i j}^{\mathrm{AM}} \gg f$ for the $\mathrm{Al} / \mathrm{InSe}$ interface. Figure 5 shows the measured and calculated phonon spectra for InSe layers with thickness $a=41 \mathrm{~nm}$ on sapphire. Four lines can be seen in the measured spectrum of the 41-nm-thick InSe layer (Fig. 5). The assumption of AM with the substrate $\left(G_{S} \gg f\right)$ reproduces the three highfrequency spectral lines. The low frequency line centered at $f=26 \mathrm{GHz}$ agrees with the calculated spectrum by assuming a lower $G_{S}$-value $\left(G_{S}=8 \mathrm{GHz}\right)$.

Figure 6 shows the measured spectra for three InSe layers with thicknesses from $a=5.4 \mathrm{~nm}$ to $a=12 \mathrm{~nm}$. We can com-

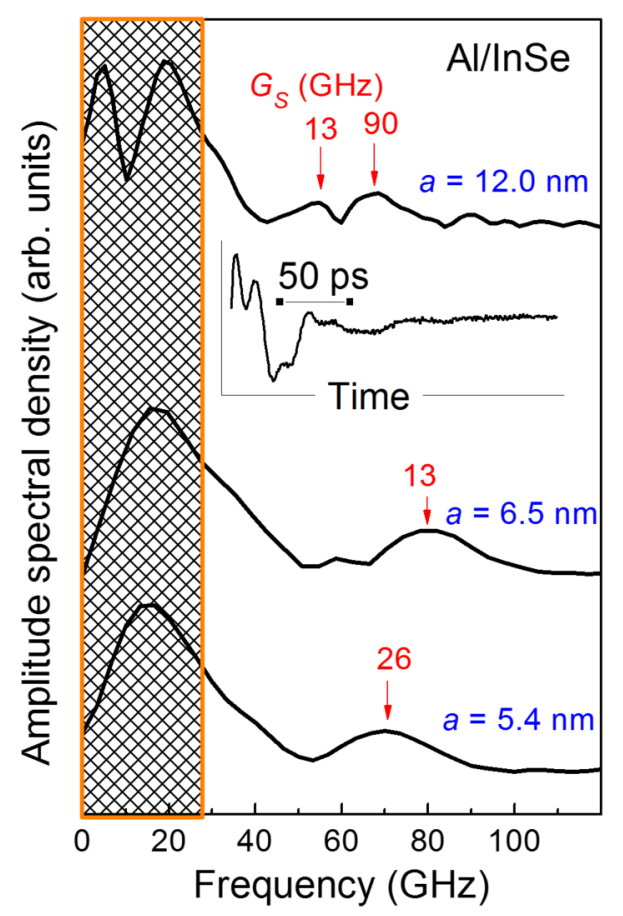

FIG. 6. Measured phonon spectra for InSe nanolayers with different thickness on a sapphire substrate. Phonons are excited via a 38-nm-thick Al-transducer. Vertical arrows indicate the frequencies of the phonon resonances obtained from the calculated spectra with corresponding values of $G_{S}$. The shaded area marks the low-frequency spectrum. The inset shows the temporal evolution of the pump-probe signals after background subtraction for the 6.5-nm-thick layer.

pare the measured resonances with the calculated ones only for $f>40 \mathrm{GHz}$. The low frequency resonances with $f \sim 20 \mathrm{GHz}$ (see shaded square in Fig. 6) have a very low quality factor $Q<1$. For these resonance modes, we may conclude only that $G_{S}>10 \mathrm{GHz}$ and that the phonon resonance frequency has weak dependence on $G_{S}$. The red vertical arrows in Fig. 6 and the values above them show the measured resonance frequencies and the corresponding $G_{S}$ values obtained from the comparison with the calculated spectra. For $a=12 \mathrm{~nm}$, two spectral lines are observed corresponding to $G_{S}=10$ and $90 \mathrm{GHz}$. For thinner layers, one spectral line is detected with values of $G_{S}$ from $13 \mathrm{GHz}(a=6.5 \mathrm{~nm})$ to $210 \mathrm{GHz}$ $(a=8 \mathrm{~nm})$.

From the data and analysis (Figs. 4 -6) of InSe layers with a wide range of thicknesses, from 5 to $100 \mathrm{~nm}$, we conclude that the coupling parameter $G_{S}$ of an InSe layer to sapphire can have values from $\sim 1 \mathrm{GHz}$ to more than $100 \mathrm{GHz}$, without any direct correlation with the InSe layer thickness. Our results also show that the measured phonon spectrum cannot always be described by a single parameter $G_{S}$. We have observed several cases when a good fit in the whole frequency range requires two or more values of $G_{S}$.

\section{B. Van der Waals heterostructures}

Figure 7 shows the results for two vdW heterostructures. The first heterostructure is a homojunction obtained by exfoliation and mechanical contact of two InSe layers of thickness 


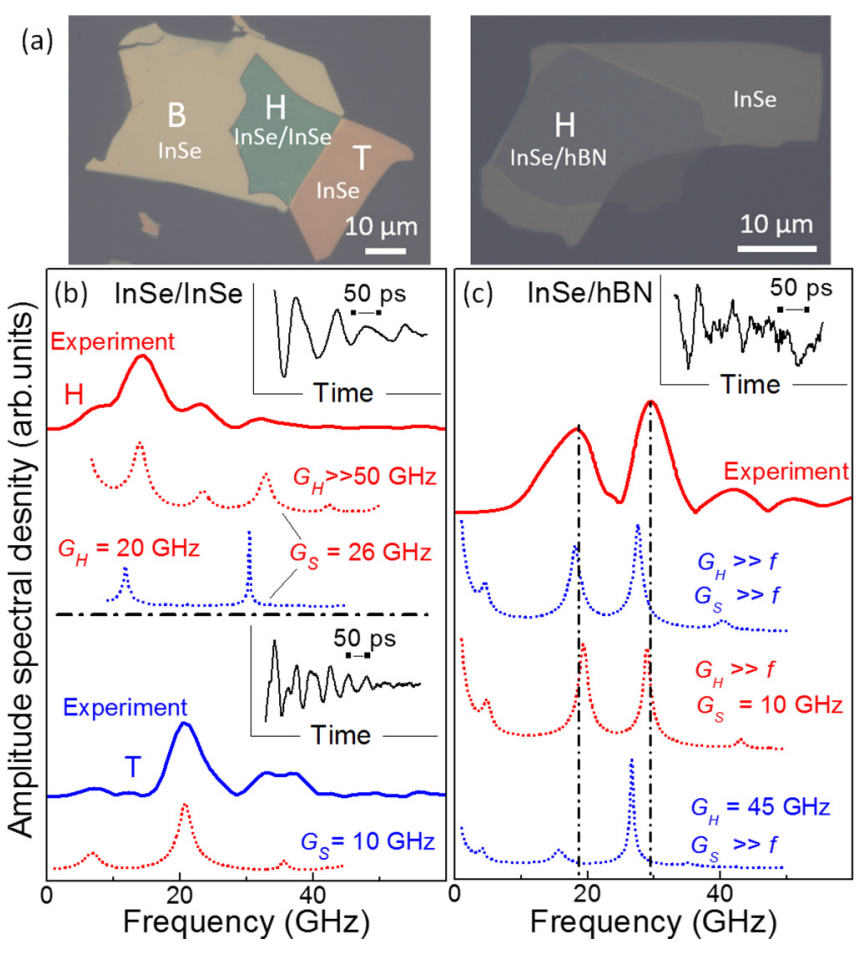

FIG. 7. (a) Optical images of an InSe/InSe homojunction (left) and of an InSe/hBN heterojunction (right). For the homojunction, $\mathrm{B}$, $\mathrm{T}$, and $\mathrm{H}$ correspond to the base, top and overlapping areas of the InSe layers, respectively. For the heterojunction, $\mathrm{H}$ corresponds to the InSe layer that is overlapped with hBN. Measured (solid lines) and calculated (dotted lines) phonon spectra for the $\operatorname{InSe}(a=77 \mathrm{~nm}) / \operatorname{InSe}(a=50 \mathrm{~nm})$ homojunction (b) and the InSe $(a=50 \mathrm{~nm}) / \mathrm{hBN}(b=81 \mathrm{~nm})$ heterojunction (c). The upper and lower panels in (b) correspond to the phonon spectra in areas $\mathrm{H}$ and $\mathrm{T}$, respectively. The theory curves are shown for different values of $G_{S}$ and/or $G_{H}$. In (b) and (c), the red dotted curves are the best theoretical fits to the measured spectra. The insets are temporal signals after background subtraction.

$a=50 \mathrm{~nm}$ and $b=77 \mathrm{~nm}$ on a sapphire substrate. The optical image of the heterostructure [see left image in Fig. 7(a)] shows three distinct areas: the area $\mathrm{H}$ of the heterostructure where the layers overlap and two regions where the top (T) and base (B) InSe layers do not overlap, thus they can be probed separately. In the experiments, we used the geometry presented in Fig. 2(c). The lower blue solid curve in Fig. 7(b) shows the phonon spectrum for the nonoverlapping area T. It reveals a peak at $f=21 \mathrm{GHz}$ in agreement with that simulated by the spring model with $G_{S}=10 \mathrm{GHz}$ [see lower blue dotted curve in Fig. 7(b)]. The red solid curve in Fig. 7(b) for the overlapping area $\mathrm{H}$ reveals a different spectrum: it consists of several lines at a regular frequency interval of $8.8 \mathrm{GHz}$. The individual frequencies agree well with those calculated for a single InSe layer with a total thickness equal to the sum of the individual layer thicknesses (e.g., $a+b=127 \mathrm{~nm}$ ) under the assumption of $G_{H} \gg 50 \mathrm{GHz}$ and $G_{S}=26 \mathrm{GHz}$. Thus we conclude that the interface between the InSe layers can be considered as elastically "ideal" for transport of coherent phonons with $f$ up to at least $50 \mathrm{GHz}$.

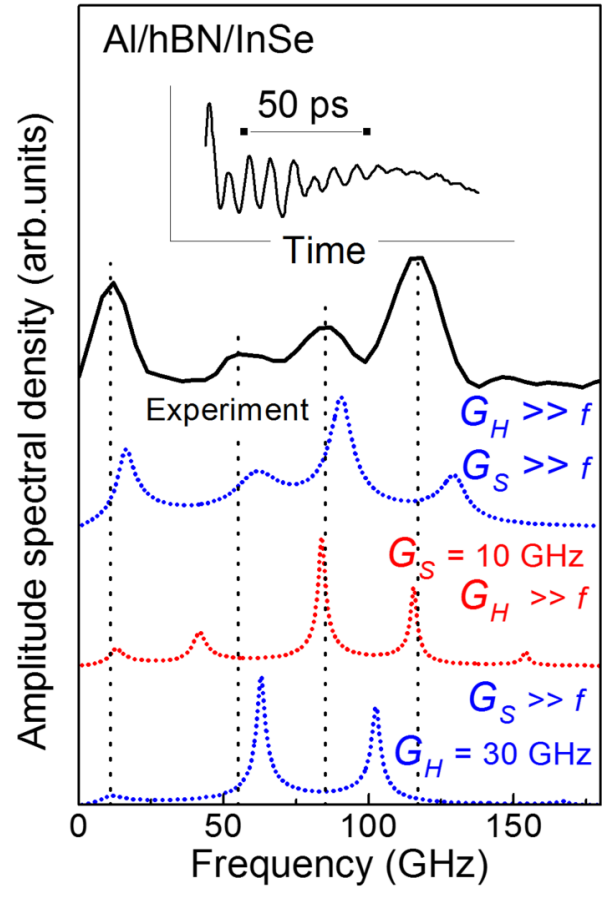

FIG. 8. Measured (solid line) and calculated (dotted line) phonon spectra for an hBN/InSe heterostructure on a sapphire substrate. The inset shows the temporal evolution of the pump-probe signals after background subtraction. The hBN and InSe layers have thickness of $a=14$ and $10 \mathrm{~nm}$, respectively. Phonons are excited from a 35-nmthick $\mathrm{Al}$ transducer. The theory curves are shown for different values of $G_{S}$ and $G_{H}$. The red dotted curve is the best theoretical fit to the experimental spectrum.

Figure 7(c) shows the results for an InSe/hBN heterostructure on a sapphire substrate measured using the transmission geometry shown in Fig. 2(a). In this heterostructure, an individual InSe nanolayer $(a=50 \mathrm{~nm})$ is stamped onto an hBN layer $(b=81 \mathrm{~nm})$ to form a heterojunction. Two resonances at $f=18$ and $30 \mathrm{GHz}$ are clearly seen in the measured spectrum indicating efficient elastic coupling between the layers. If there was no good elastic contact between the layers, the spectrum would reveal only the nanomechanical resonances excited in the top InSe layer. In fact, the underlying hBN layer cannot be excited directly as it is transparent at the wavelength of the pump probe. As for the case of the InSe/InSe homojunction, also in this case the calculated spectrum indicates acoustic mismatch at the InSe/hBN vdW heterointerface [see dotted lines in Fig. 7(c)]. The best agreement between our model with the experiment is obtained for $G_{H} \gg 30 \mathrm{GHz}$ and $G_{S}=$ $10 \mathrm{GHz}$ [Fig. 7(c), red dotted line].

Similar results were obtained for other vdW heterostructures and experimental geometries. For example, Fig. 8 shows the results for an InSe/hBN heterostructure on sapphire with thicknesses of the hBN and InSe layers of $a=14 \mathrm{~nm}$ and $b=10 \mathrm{~nm}$, respectively. Coherent phonons were injected into the heterostructure from an Al-film (thickness of $35 \mathrm{~nm}$ ) evaporated on top of the hBN layer [experimental scheme Fig. 2(d)]. From the observation of four distinct spectral lines in the frequency range up to $150 \mathrm{GHz}$ and the comparison with the calculated phonon spectra (dotted lines in Fig. 8), we 
conclude that there is a strong coupling at the heterointerface. The best agreement is obtained assuming $G_{H} \gg 100 \mathrm{GHz}$ and $G_{S}=10 \mathrm{GHz}$. If we assume that the coupling at the $\mathrm{vdW}$ heterointerface drops to $G_{H}=30 \mathrm{GHz}$ (see the lowest dotted curve in Fig. 8), the spectrum would show only two resonances.

In summary, the measured spectra of coherent phonons reveal nanomechanical resonances with frequency, amplitude, and spectral width that depend on the strength of elastic coupling between the different layers and/or with the supporting substrate. Our study of the acoustic mismatch between vdW layers in InSe/InSe homojunctions and InSe/hBN heterojunctions reveal an ideal interface and strong coupling over a wide range of frequencies up to $\sim 100 \mathrm{GHz}$.

\section{DISCUSSION}

In this section, we examine how the frequency and the finesse of the phonon resonances depend on the elastic properties of the nanolayers and vdW heterostructures, and on phonon scattering at interfaces, i.e., the interface between two vdW layers or the interface between a vdW layer and its supporting substrate.

\section{A. Elastic properties of InSe and hBN}

The layered crystal structure of InSe and hBN causes anisotropic elastics properties, e.g., elastic constant tensor $c_{i j}$ and sound velocity $s$. For our experiments with propagation of coherent phonons in the direction $x$ perpendicular to the $\mathrm{vdW}$ layer planes, the sound velocity is calculated as $s=\sqrt{c_{33} / \rho}$ (for values see Table I) using elastic parameters measured in bulk crystals by standard ultrasonics $[64,75]$ and Brillouin scattering [76].

To assess whether the use of elastic parameters for bulk crystals is a valid assumption to interpret our findings, we derive elastic parameters from an empirical LennardJones potential $U(x)$ for the vdW interaction between the layers:

$$
U(x)=E_{B}\left[\left(\frac{x_{0}}{x}\right)^{12}-2\left(\frac{x_{0}}{x}\right)^{6}\right],
$$

where $x_{0}$ is the distance between the edges of the atomic monolayers and $E_{B}$ is their binding energy. In hBN, $x_{0}$ corresponds to the interlayer period, e.g., $x_{0}=c \sim 0.7 \mathrm{~nm}$. In InSe, each vdW monolayer consists of four covalently ionic bonded atomic sheets (Se-In-In-Se) of thickness $0.53 \mathrm{~nm}$. The distance between Se-atoms at the boundaries of each $\mathrm{vdW}$ layer is $x_{0} \sim 0.38 \mathrm{~nm}[77,78]$. The value of $E_{B}$ for $\mathrm{hBN}$ is $E_{B}=13 \mathrm{meV} / \AA^{2}$ [79]; also, vdW interactions in InSe were found to be as weak as in graphite and other vdW crystals [78], for which $E_{B}$ is in the range 13 to $21 \mathrm{meV} / \AA^{2}$ [79].

For a small change $\Delta x$ in the equilibrium separation of the vdW layers, i.e., $\Delta x=x-x_{0} \ll x_{0}$, Eq. (10) can be written as

$$
U(\Delta x)=36 E_{B} x_{0}^{-2}(\Delta x)^{2},
$$

from which we derive the specific stiffness of the $\mathrm{vdW}$ coupling:

$$
\eta_{0}=72 E_{B} x_{0}^{-2} .
$$

Alternatively, we can estimate the value of $\eta_{0}$ from the elastic constant of the bulk crystals:

$$
\eta_{0}=c_{33} / c \text {. }
$$

Using Eqs. (12) and (13) for $\mathrm{hBN}$, we derive $\eta_{0}=3.1 \times$ $10^{19} \mathrm{~N} / \mathrm{m}^{3}$ and $\eta_{0}=3.7 \times 10^{19} \mathrm{~N} / \mathrm{m}^{3}$, respectively. Thus different models give similar stiffnesses for $\mathrm{hBN}$. Also, using Eq. (13) and the values for $c_{33}$ and $x_{0}$ for InSe (see Table I), we derive $\eta_{0}=4.5 \times 10^{19} \mathrm{~N} / \mathrm{m}^{3}$. From this value of $\eta_{0}$ and Eq. (12), we estimate that $E_{B}=15 \mathrm{meV} / \AA^{2}$ for InSe, within the range of values of $E_{B}$ reported for other vdW crystals [80].

We use the same "spring" model approach to describe the elastic coupling between two materials (Sec. IIIB, and Appendix) and calculate the coupling parameter $G_{0}$ between two vdW layers. Using the estimated values of $\eta_{0}$, we obtain $G_{0} \sim 1.0$ and $1.5 \mathrm{THz}$ for InSe and hBN, respectively. These values are smaller than the zone edge phonon frequencies for propagation perpendicular to the layers, i.e., $f=1.2$ and 2.4 THz for InSe [81] and hBN [75], respectively, but much higher than the maximum phonon frequency $f \sim 100 \mathrm{GHz}$ measured in our experiments. Thus we conclude that our use of the continuous approximation with bulk elastic parameters and sound velocity of InSe and hBN is valid in our analysis of the phonon spectra.

\section{B. Elastic properties of interfaces}

In the analysis of phonon transport, the elastic properties of interfaces are as important as the elastic properties of the nanolayers themselves. The interface defines the absolute value $|R|$ and phase shift $\Delta \varphi$ of the specular phonon reflection at the interface. The value of $\Delta \varphi$ varies from 0 to $\pi$ depending on the elastic coupling. This phase shift governs the interference of coherent phonons and hence defines the frequency $f_{n}$ of the phonon resonances.

We use a phenomenological spring model to describe the interface between two materials (see Sec. III and Appendix). In this model, the interface is described as a weightless spring (Fig. 3) with a frequency independent specific stiffness $\eta$. As shown in Sec. IV, the value of $f_{n}$ measured in several layers and heterostructures agrees well with acoustic mismatch $(G \gg$ $f_{n}$ ), thus indicating a good elastic contact at the interface over a wide frequency range. However, in some cases, we have to assume a weak coupling between a layer and its supporting substrate $\left(G_{S} \sim f_{n}\right)$ and/or different values of $G_{S}$. To interpret these results, we examine the morphology of the interfaces.

The elastic coupling between the exfoliated nanolayers depends on the quality of the surfaces, which can be nonuniform over the area $\left(5 \times 5 \mu \mathrm{m}^{2}\right)$ of the probing spot. Figure 9 shows the AFM images and $x$ profiles for an InSe layer on a sapphire substrate. The AFM data show that the roughness of the InSe surface (up to $1 \mathrm{~nm}$ ) is significantly larger than for the sapphire substrate $(\sim \pm 0.1 \mathrm{~nm})$. Also, it is larger than that of hBN, which has an abrupt and atomically smooth surface $[82,83]$. Thus the most likely reason for the imperfectness 

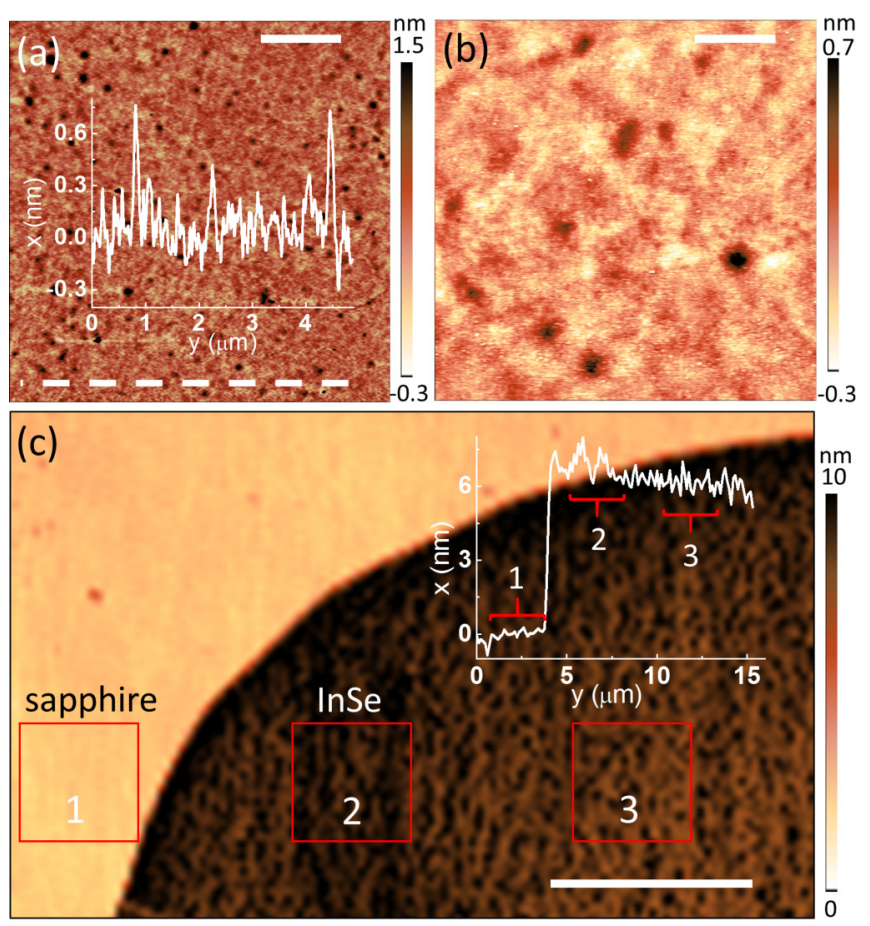

FIG. 9. (a) Atomic force microscopy (AFM) image of a $5 \mu \mathrm{m} \mathrm{x}$ $5 \mu \mathrm{m}$ region of InSe (scale bar $=1 \mu \mathrm{m}$ ). Inset: AFM profile along the white dotted line. (b) AFM image of a $1 \mu \mathrm{m} \times 1 \mu \mathrm{m}$ region of InSe (scale bar $=0.2 \mu \mathrm{m}$ ). (c) AFM image of an InSe nanolayer on a sapphire substrate. (Inset) AFM profile along a line through regions marked by squares 1-2-3. These areas show regions with different surface roughness (scale bar $=5 \mu \mathrm{m}$ ).

of the interface revealed in our experiments is the surface roughness of the InSe layer. This also implies inhomogeneities in the coupling parameter $\eta_{S}$ and $\eta_{H}$. Two atomically flat $\mathrm{vdW}$ layers interact elastically via vdW forces and have a binding energy $E_{B} \sim 10 \mathrm{meV} / \AA^{2}$, which does not depend strongly on the specific material [78,79]. The value of $E_{B}$ gives a stiffness $\eta_{0} \sim 10^{19}-10^{20} \mathrm{~N} / \mathrm{m}^{3}$ and corresponding coupling parameter $G_{0} \sim 10^{3} \mathrm{GHz}$. Due to the strong dependence of the $\mathrm{vdW}$ potential energy on distance [Eq. (10)], the increase of $x$ by only one layer of atoms due an imperfect interface results in the decrease of $\eta_{0}$ and $G_{0}$ by almost two orders of magnitude $(G \sim 10 \mathrm{GHz})$, leading to changes in the complex phonon reflectivity $R$ [Eq. (A9)] and phonon resonance frequency $f_{n}$. Thus, for sub-THz phonons, the areas of atomically perfect interface behave as in the case of AM while the increase of the distance at the interface of just one atomic layer results in the reflection of sub-THz phonons, as for the case of a free surface.

To characterize the coherent phonon resonances, we consider two types of regions. The first type, labeled "AM," has an atomically flat interface: the distance $x$ between the layers is uniform with a coupling parameter $G_{\mathrm{AM}} \sim 10^{3} \mathrm{GHz}$. The second type corresponds to a "free surface" (FS): the distance between the layers is $x \geqslant 2 x_{0}$ with a coupling parameter $G_{\mathrm{FS}} \leqslant f$. It is important to compare the average size of the AM and FS regions, $\overline{L_{\mathrm{AM}}}$ and $\overline{L_{\mathrm{FS}}}$, respectively, with the phonon wavelength $\Lambda$. For InSe, the value of $\Lambda=s / f$ varies from $250 \mathrm{~nm}$ down to $25 \mathrm{~nm}$ for $f$ in the range $10-100 \mathrm{GHz}$. In the assumption that the correlation length is small compared with $\Lambda$ we may consider $\overline{L_{\mathrm{AM}}}$, and $\overline{L_{\mathrm{FS}}} \ll \Lambda$, and then for the phonon resonances observed in our experiments the interface roughness is averaged over $\Lambda$. In this case, we can characterize the interface using a single coupling parameter:

$$
G=G_{0} \frac{S_{\mathrm{AM}}}{S_{\mathrm{AM}}+S_{\mathrm{FS}}},
$$

where $S_{\mathrm{AM}}$ and $S_{\mathrm{FS}}$ are the areas of the AM and FS regions within the probe spot. We refer to this type of interface as "mono." For $S_{\mathrm{AM}} \gg S_{\mathrm{FS}}$, we have $G=G_{0} \sim 1 \mathrm{THz}$ and the interface may be considered as elastically perfect and the AM model valid for all acoustic phonons, provided that the phonon dispersion is not important. An alternative case is that of a "broken" interface, i.e., $S_{\mathrm{AM}} \ll S_{\mathrm{FS}}$ and hence $G \ll G_{0}$. In general, the interface may have a more complicated morphology. For instance, in the area of the probe spot, there may be areas with different mean values of $G$. Examples of areas of InSe with different surface roughness and hence different $G$ are shown in Fig. 9(c) by squares labelled 2 and 3. Their typical in plane size $(\sim 1 \mu \mathrm{m})$ is larger than $\Lambda$. The corresponding profiles in Fig. 9(c) of these areas demonstrate that the roughness of the surface in area 2 is higher than in area 3, thus leading to $G_{3}>G_{2}$ and a "dual" interface. In general, we can identify both "mono" and "dual" interfaces for InSe layers on a sapphire substrate.

In contrast, we find that the interface between two vdW layers (e.g., the interface in $\mathrm{InSe} / \mathrm{InSe}$ and $\mathrm{InSe} / \mathrm{hBN}$ ) is elastically perfect within the measured frequency range. The comparison between the experimental and calculated spectra in Figs. 7 and 8 indicates that the phonon frequency would be very different if the layers were not elastically coupled. Thus we may exclude the case of weak coupling $\left(G_{H} \leqslant f\right)$ at the interface between two vdW layers in all our heterostructures. Also, we can explain the phonon spectra using the acoustic mismatch model over a wide frequency range up to $120 \mathrm{GHz}$ (Fig. 8).

At a first glance, it may seem surprising that the interface of $\mathrm{InSe} / \mathrm{InSe}$ and InSe/hBN heterostructures has a stronger elastic coupling compared to the InSe/sapphire interface since the surface roughness of the InSe layer is the same in both cases. To explain this behavior, we note that there is a difference in acoustic impedances between InSe and sapphire: $Z_{\text {sapphire }}=$ $3.2 Z_{\text {InSe }}$. Thus the value of $G_{0}$ for the interface of InSe with sapphire is noticeably smaller than for InSe/InSe and InSe/hBN interfaces [see Eqs. (7) and (8)]. Also, the more rigid sapphire substrate may not be able to adapt to the InSe surface compared to the case of two van der Waals layers.

In summary, we have shown that the frequencies of the phonon resonances are dependent on both the thickness of the layer and elastic coupling at the interface. The latter factor was not considered in earlier works [55-57] where it was assumed that the nanolayer has either a perfect elastic contact with the substrate [57] or behaves as a suspended film [55]. In our work, we show that the elastic properties of vdW interfaces can be probed by sub-THz coherent phonons. None of the other existing measuring techniques can access this information. In particular, some of the available microscopy techniques, such as TEM, examine only static surface properties, whereas 
others, such as AFM, can only probe surfaces and/or lowfrequency elastic properties.

\section{Scattering and decay of coherent phonons}

An important characteristic of a phonon resonance is its linewidth $\Delta f \sim\left(2 \tau^{*}\right)^{-1}$, where $\tau^{*}$ is the phonon decay time. The measured linewidths indicate $\tau^{*} \leqslant 50 \mathrm{ps}$. Different mechanisms can contribute to $\tau^{*}$, e.g.,

$$
\left(\tau^{*}\right)^{-1}=\left(\tau_{S}\right)^{-1}+\left(\tau_{A}\right)^{-1}+\left(\tau_{I}\right)^{-1}+\left(\tau_{D}\right)^{-1}+\left(\tau_{R}\right)^{-1},
$$

where $\tau_{S}$ is the time of the diffusive scattering at interfaces and free surfaces; $\tau_{A}$ is the anharmonic decay time; $\tau_{I}$ is the effective time related to inhomogeneities in film thickness; $\tau_{D}$ describes the decay of the laterally localized resonance due to acoustic diffraction, and $\tau_{R}$ describes the phonon decay time due to energy emission into the substrate. Only the latter one is included in our previous theoretical analysis and can be expressed as

$$
\tau_{R}=-\frac{2 a}{s \ln |R|},
$$

where $s$ is the sound velocity of the nanolayer and $a$ is the layer thickness. Using Eq. (3) for $\operatorname{AM}\left(G_{S} \gg f\right)$ and for an InSe nanolayer layer with $a \sim 100 \mathrm{~nm}$ on a sapphire substrate, we estimate $\tau_{R} \sim 100 \mathrm{ps}$, which is about three times longer than the value $\tau^{*} \sim 30$ ps derived from the measured phonon spectra (see lower spectrum in Fig. 4). The calculated value of $\tau_{R}$ increases for weak coupling and correspondingly small values of $G_{S}$. Thus the difference between the measured and calculated $\tau_{R}$ is due to other decay mechanisms, which are discussed below.

The value of $\tau_{A}$ is governed by third-order elastic constants, which are not known for the studied vdW layers. We may assume that $\tau_{A}$ is not much different from the values found in other crystalline materials for which $\tau_{A}>1 \mathrm{~ns}$ at frequencies below $100 \mathrm{GHz}$ [84]. This time is significantly longer than the measured time and thus it will be not considered further.

The value of $\tau_{D}$ is governed by the lateral dimension $L_{G}$ of the local AM and FS resonators characterized by an effective rigidity $G$ at the interfaces in Eq. (14). The value of $\tau_{D}$ can be estimated as the time taken for an acoustic wave to travel a diffraction length $L_{D}$, e.g., $\tau_{D} \approx L_{D} / s \approx\left(L_{G}^{2} / \Lambda\right) / s$, where $L_{G}$ is the diameter of the acoustic beam. Since $L_{G} \gg \Lambda, \tau_{D}$ is much longer than the measured time. For example, for phonons with $\Lambda \sim 100 \mathrm{~nm}$ in an InSe film strongly coupled to sapphire, $\tau_{D}$ becomes equal to the measured time when $L_{G} \sim \Lambda$. Thus the losses due to diffraction are not important only for the local resonators with $L_{G}$ significantly exceeding the acoustic wavelength $\left(L_{G} \gg \Lambda\right)$.

The decay time $\tau_{I}$ could be caused by inhomogeneous broadening of the resonance spectral line due to the long-scale ( $\sim 1 \mu \mathrm{m}$ in the plane of the nanolayers) thickness variations of the InSe and hBN nanolayers. The AFM profiles of our layers show variations in the thickness of $\pm 10 \%$. Thus $\tau_{I}$ can represent a significant contribution to the measured lifetime. For example, the measured lifetime $\tau^{*} \sim 80$ ps for $13 \mathrm{GHz}$ phonons in an InSe nanolayer weakly coupled to sapphire (upper spectrum in Fig. 4) could be assigned to $\pm 15 \%$ variations in the InSe film thickness.

Finally, the decay time $\tau_{S}$ in Eq. (15) accounts for the losses caused by diffusive scattering at an interface due to their roughness, point defects, monolayer steps and/or boundaries between regions with different $G$. The AFM image in Fig. 9 shows variations of the InSe layer thickness over length scales $>100 \mathrm{~nm}$. We examine their effect on acoustic wave scattering using the small slope approximation (SSA) [85,86]. For our estimate of $\tau_{S}$, we use the dependence of the specular scattering probability (SSP) on the root-mean square roughness $\sigma_{\text {rms }}$ of the surface [87]. The data presented in Ref. [87] for $117-\mathrm{GHz}$ phonons in $\mathrm{GaN}$ can be applied to estimate $\tau_{S}$ for $\sim 20 \mathrm{GHz}$ phonons in InSe films as both systems have similar phonon wavelengths $(\sim 70 \mathrm{~nm})$; also, the correlation length of the surface roughness of InSe ( 10-40 nm measured by AFM) and in $\mathrm{GaN}$ ( $\sim 24-38 \mathrm{~nm}$ in Ref. [87]) are similar. For values of $\tau_{S}$ equal to the measured ones, the SSP is $\approx 0.15$, implying $\sigma_{\mathrm{rms}} \approx 8 \mathrm{~nm}[87]$. This value exceeds by an order of magnitude the value $\sigma_{\text {rms }} \sim 1 \mathrm{~nm}$ for the InSe surface (see AFM data in Fig. 9). Thus we may conclude that diffusive scattering of $\sim 20$ $\mathrm{GHz}$ phonons at the interfaces with $\sigma_{\text {rms }} \leqslant 1 \mathrm{~nm}$ is negligible and, therefore, its contribution to $\tau_{S}$ can be ignored.

In summary, from the comparison of the measured and calculated spectra, we find that the measured spectral widths $\Delta f_{n} \geqslant 10 \mathrm{GHz}$ exceed those calculated by acoustic mismatch by a factor of 2 . In accordance with the estimates presented above, this difference should be mostly attributed to the inhomogeneous broadening of the spectral line.

\section{CONCLUSIONS AND OUTLOOK}

We have used picosecond ultrasonics to measure the coherent acoustic phonon spectra of InSe layers with thicknesses from 5 to $\sim 100 \mathrm{~nm}$ and their heterostructures. The spectra consist of several well separated lines in the frequency range from $\sim 10$ to $\sim 100 \mathrm{GHz}$, revealing the existence of nanomechanical resonances in all layers due to phonon quantization in the direction perpendicular to the layer plane. The frequencies of quantized phonons depend not only on the layer thickness, but also on the elastic coupling parameters at the interfaces. The values of the resonance frequencies measured in the present work agree well with those derived from the continuous elastic theory using bulk elastic constants and effective springs with frequency independent stiffnesses. In some cases, the inhomogeneities of the elastic coupling at the interfaces should also be taken into the account to explain the phonon spectrum.

Our data and analysis indicate that the vdW interface of InSe/InSe and InSe/hBN heterostructures can be described by the acoustic mismatch approach and considered as elastically ideal for phonon frequencies up to at least $150 \mathrm{GHz}$. In contrast, the elastic coupling of InSe layers to a substrate may not always be ideal and the phonon resonances may not always be described by a single elastic coupling parameter.

The present work focuses on elastic properties of interfaces rather than phonon properties of the layers, as reported in earlier works [55-58]. Furthermore, our conclusions are broader than those in the earlier work by Beardsley et al. [58], which reported InSe layers weakly coupled to a substrate. In the present work, we show that the elastic coupling of a vdW 
nanolayer to its supporting substrate may vary and can be described by the spring model; also, in both InSe/InSe and InSe/hBN vdW heterostructures, we observe a strong elastic coupling, well described by the acoustic mismatch model. Means of probing phonon transport across an interface can provide the foundation for designing and exploiting new materials and quantum systems: the strength of coupling between the layers is critical to both charge and phonon transport and thus pivotal to the future development of functional devices, including new device concepts for high-frequency electronics and thermoelectrics. The spectrum of nanomechanical resonances changes significantly when the distance between the layers changes by only one layer of atoms. Thus it could be used to probe and manipulate interfaces with an unprecedented spatial resolution and over a wide frequency range. To date, the only technique that could offer such spatial resolution is TEM, but this is time-consuming and requires special sample preparation to expose interfaces to the probing electron beam. On the other hand, it should be possible to use a focused laser beam to generate coherent phonons as it scans over the sample to measure the spectrum and lifetime of the phonons in the structures or the spectrum of phonons emitted into the substrate.

Finally, of particular interest is the possibility of using strain to modulate the electronic properties for high-frequency acoustoelectric and acousto-optic devices. In particular, 2D InSe exhibits high mechanical flexibility, can sustain high mechanical strain $(>20 \%)$ and its electronic properties can change under both uniaxial and biaxial compressive strains [88]. These properties, which have been only recently predicted by theory [88], are highly desirable for new "straintronic" devices and sub-THz nano-electro-mechanical and optomechanical devices. In particular, thin layers and heterostructures weakly coupled to the substrate may have high finesse $(>10)$ and used as sensors for single molecules adsorbed at surfaces and interfaces. Further studies may involve the study of single atomic layers and correspondingly $\mathrm{THz}$ phonons. In this case, the approximation of continuous media and acoustic mismatch approach may not be valid. There are no principle limitations to these studies, which will contribute to important advances in the physics of phonons and their exploitation in novel 2D structures.

\section{ACKNOWLEDGMENTS}

This work was supported by the Engineering and Physical Sciences Research Council [Grant No. EP/M012700/1] (EPSRC); the EU FP7 Graphene Flagship Project 604391; The Leverhulme Trust; and the National Academy of Sciences of Ukraine. K.W. and T.T. acknowledge support from the Elemental Strategy Initiative conducted by the MEXT, Japan.

\section{APPENDIX}

For propagation of longitudinal acoustic phonons along the $x$ direction, perpendicular to the plane of the vdW layers, the elastic equation is

$$
\rho_{i} \frac{\partial^{2} u_{i}}{\partial^{2} t}=c_{i} \frac{\partial^{2} u_{i}}{\partial^{2} x}+\frac{\partial \sigma_{0}}{\partial x},
$$

where the index $(i)$ defines the vdW layer or the substrate $(s)$ with density $\rho_{i}$ and elastic constant $c_{i}$, and $\sigma_{0}$ is the stress generated by the optical pump pulse. In our experiments, the pump pulse is absorbed in a thin InSe layer or $\mathrm{Al}$ transducer and with good approximation we may consider the excitation as spatially homogeneous due to fast transport of photoexcited carriers and heat in the layer [69]. Then $\sigma_{0}$ does not depend on $x$ except at the interfaces at which the second term in the right part of Eq. (A1) is equal to zero. For comparison with the experiment, it is convenient to present the results in a spectral domain for the Fourier components of displacement $\tilde{u}(\omega, x)$ and strain $\tilde{\varepsilon}(\omega, x)=d \tilde{u} / d x$. In the spectral domain, Eq. (A1) may be written as

$$
\frac{d^{2} \tilde{u}_{i}(\omega, x)}{d x^{2}}+\frac{\omega^{2}}{s_{i}^{2}} \tilde{u}_{i}(\omega, x)=0,
$$

where $s_{i}=\sqrt{c_{i} / \rho_{i}}$ is the sound velocity in the $i$ th layer or substrate. The general solution of Eq. (A2) for phonons in $\mathrm{vdW}$ layers is given by Eqs. (1) and (2) in Sec. III.

In the acoustic mismatch model, the displacement and stress are continuous at each interface. An example for an InSe/hBN heterostructure on a substrate is schematically shown in Fig. 3(a). There are two vdW nanolayers with thicknesses $a$ (InSe, layer 1) and $b(\mathrm{hBN}$, layer 2) deposited on a sapphire substrate $(s)$. There are three interfaces at $x=-a, x=0$, and $x=b$. The stress $\sigma_{0}$ induced by the optical pump pulse is generated only in the InSe nanolayer. In contrast, $\sigma_{0}=0$ in the hBN layer and the sapphire substrate, which do not absorb the pump light at $\lambda=400 \mathrm{~nm}$. The boundary conditions may be written as

$$
\begin{aligned}
& A_{1} k_{1} \sin \left(k_{i} x\right)+B_{1} k_{1} \cos \left(k_{i} x\right)+\widetilde{\sigma_{0}}=0 \text { at } x=-a \text {, } \\
& \left.\begin{array}{rl}
A_{1} & =A_{2} \\
B_{1} c_{1} k_{1}+\tilde{\sigma}_{0} & =B_{2} c_{2} k_{2}
\end{array}\right\} \text { at } x=0,
\end{aligned}
$$

and

$$
\left.\begin{array}{c}
A_{2} \cos \left(k_{1} b\right)+B_{2} \sin \left(k_{2} b\right)=A_{s} \exp \left(-i k_{s} b\right) \\
-A_{2} c_{2} k_{2} \sin \left(k_{2} b\right)+B_{2} c_{2} k_{2} \cos \left(k_{2} b\right) \\
=-i A_{s} c_{S} k_{s} \exp \left(-i k_{s} b\right)
\end{array}\right\} \text { at } x=b .
$$

Solving Eqs. (A3), (A4), and (A5), we find five complex amplitudes $A_{1}, A_{2}, B_{1}, B_{2}$, and $A_{S}$, and substituting them into Eqs. (1) and (2), we get the Fourier spectrum of coherent phonons at the coordinate $x$.

The spring model describes the elastic properties of an interface that is not ideal. In this model, the neighboring boundaries of two materials are considered to be connected by springs with specific stiffness $\eta$ [see Figs. 3(b) and 3(c)]. The parameter $\eta$ characterizes the force $\Delta F$ per unit area, which appears during the modulation of the distance $\Delta x$ between the boundaries of the neighboring materials. In the linear approximation, according to Hooke's law, we can write

$$
\Delta F=\eta \Delta x .
$$


The boundary conditions for stress at the free surface, $x=$ $-a$, are the same as Eq. (A3). For the interface at $x=0$ and $x=b$, the conditions for the continuity of the stress can be written, respectively, as

$$
\begin{aligned}
B_{1} c_{1} k_{1}+\tilde{\sigma}_{0} & =\eta_{H}\left(A_{2}-A_{1}\right) \\
B_{2} c_{2} k_{2} & =\eta_{H}\left(A_{2}-A_{1}\right),
\end{aligned}
$$

and

$$
\left.\begin{array}{rl}
- & A_{2} c_{2} k_{2} \sin \left(k_{2} b\right)+B_{2} c_{2} k_{2} \cos \left(k_{2} b\right) \\
= & \eta_{S}\left[A_{s} \exp \left(-i k_{s} b\right)-A_{2} \cos \left(k_{2} b\right)-B_{2} \sin \left(k_{2} b\right)\right] \\
& -A_{s} i c_{3} k_{3} \exp \left(-i k_{3} b\right) \\
= & \eta_{S}\left[A_{s} \exp \left(-i k_{s} b\right)-A_{2} \cos \left(k_{2} b\right)-B_{2} \sin \left(k_{2} b\right)\right]
\end{array}\right\} .
$$

Equations (A7) and (A8) give the solution for coefficients $A_{i}$ and $B_{i}$.
For strain waves incident from $i$ to $j$, the equation for the reflection coefficient at the interface is

$$
R_{i j}=\frac{\eta_{i j}\left(Z_{j}-Z_{i}\right)-i Z_{j} Z_{i} \omega}{\eta_{i j}\left(Z_{j}+Z_{i}\right)+i Z_{j} Z_{i} \omega}=\frac{G_{i j} R_{i j}^{\mathrm{AM}}-i f}{G_{i j}+i f},
$$

where $R_{i j}^{\mathrm{AM}}$ is the reflection coefficient given by Eq. (3). For $G_{i j}=0, R_{i j}=-1$ as for a free surface. For $G_{i j} R_{i j}^{\mathrm{AM}} \gg f$, $R_{i j} \approx R_{i j}^{\mathrm{AM}}$. In contrast to the scenario of AM, in this case $R_{i j}$ is complex, thus implying that the phase change at the interface depends on $\eta$. This is also responsible for the difference in the values of $f_{n}$ in the spring and AM models, and the dependence of $f_{n}$ on $\eta$. In particular, an important difference between the spring and AM models is that $R_{i j}$ depends on $f$ in the spring model while $R_{i j}$ is the same for all frequencies for AM. Thus it may happen that for constant values of $\eta$, the fundamental resonance mode with $n=1$ behaves as in the case of AM while for higher modes, $f_{n}$ is close to the spectrum for free standing films. The spring model is further complicated if the spring stiffness depends on $f$ [89] or if springs have finite mass [90], which is not considered in this work.
[1] O. Matsuda, M. C. Larciprete, R. L. Voti, and O. B. Wright, Fundamentals of picosecond laser ultrasonics, Ultrasonics 56, 3 (2015).

[2] S. Volz, J. Ordonez-Miranda, A. Shchepetov, M. Prunnila, J. Ahopelto, T. Pezeril, G. Vaudel, V. Gusev, P. Ruello, E. M. Weig et al., Nanophononics: state of the art and perspectives, Eur. Phys. J. B 89, 15 (2016).

[3] G. Tas, R. J. Stoner, H. J. Maris, G. W. Rubloff, G. S. Oehrlein, and J. M. Halbout, Noninvasive picosecond ultrasonic detection of ultrathin interfacial layers: $\mathrm{CF}_{x}$ at the $\mathrm{Al} / \mathrm{Si}$ interface, Appl. Phys. Lett. 61, 1787 (1992).

[4] C. Rossignol, B. Perrin, B. Bonello, P. Djemia, P. Moch, and H. Hurdequint, Elastic properties of ultrathin permalloy/alumina multilayer films using picosecond ultrasonics and Brillouin light scattering, Phys. Rev. B 70, 094102 (2004).

[5] T. Pezeril, N. Chigarev, D. Mounier, S. Gougeon, P. Ruello, J.-M. Breteau, P. Picart, and V. Gusev, Lumped oscillations of a nanofilm at adhesion bond, Eur. Phys. J. Special Topics 153, 207 (2008).

[6] I. J. Chen, P. A. Mante, C. K. Chang, S. C. Yang, H. Y Chen, Y. R. Huang, L. C. Chen, K. H. Chen, V. Gusev V, and C. K. Sun, Graphene-to-substrate energy transfer through out-of-plane longitudinal acoustic phonons, Nano Lett. 14, 1317 (2014).

[7] A. Amziane, L. Belliard, F. Decremps, and B. Perrin, Ultrafast acoustic resonance spectroscopy of gold nanostructures: Towards a generation of tunable transverse waves, Phys. Rev. B 83, 014102 (2011).

[8] C. Jean, L. Belliard, T. W. Cornelius, O. Thomas, Y. Pennec, M. Cassinelli, M. E. Toimil-Molares, and B. Perrin, Spatiotemporal imaging of the acoustic field emitted by a single copper nanowire, Nano Lett. 16, 6592 (2016)

[9] Y. Guillet, B. Audoin, M. Ferrié, and S. Ravaine, All-optical ultrafast spectroscopy of a single nanoparticle-substrate contact, Phys. Rev. B 86, 035456 (2012).
[10] R. Legrand, A. Huynh, B. Jusserand, and B. Perrin, and A. Lemaître, Direct measurement of coherent subterahertz acoustic phonons mean free path in GaAs, Phys. Rev. B 93, 184304 (2016).

[11] D. M. Moss, A. V. Akimov, B. A. Glavin, M. Henini, and A. J. Kent, Ultrafast Strain-Induced Current in a GaAs Schottky Diode, Phys. Rev. Lett. 106, 066602 (2011).

[12] E. S. K. Young, A.V. Akimov, M. Henini, L. Eaves, and A. J. Kent, Subterahertz Acoustical Pumping of Electronic Charge in a Resonant Tunneling Device, Phys. Rev. Lett. 108, 226601 (2012).

[13] C. L. Poyser, A. V. Akimov, R. P. Campion, and A. J. Kent, Coherent phonon optics in a chip with an electrically controlled active device, Sci. Rep. 5, 8279 (2015).

[14] K. Shinokita, K. Reimann, M. Woerner, T. Elsaesser, R. Hey, and C. Flytzanis, Strong Amplification of Coherent Acoustic Phonons by Intraminiband Currents in a Semiconductor Superlattice, Phys. Rev. Lett. 116, 075504 (2016).

[15] C. L Poyser, A. V. Akimov, A. G. Balanov, R. P. Campion, and A. J. Kent, A weakly coupled semiconductor superlattice as a harmonic hypersonic-electrical transducer, New J. Phys. 17, 083064 (2015).

[16] V. V. Temnov, Ultrafast acousto-magneto-plasmonics, Nat. Photonics 6, 728 (2012).

[17] C. Brüggemann, A. V. Akimov, B. A. Glavin, V. I. Belotelov, I. A. Akimov, J. Jäger, S. Kasture, A. V. Gopal, A. S. Vengurlekar, D. R. Yakovlev, A. J. Kent, and M. Bayer, Modulation of a surface plasmon-polariton resonance by subterahertz diffracted coherent phonons, Phys. Rev. B 86, 121401 (2012).

[18] K. O’Brien, N. D. Lanzillotti-Kimura, J. Rho, H. Suchowski, $\mathrm{X}$. Yin, and X. Zhang, Ultrafast acousto-plasmonic control and sensing in complex nanostructures, Nat. Commun. 5, 4042 (2014). 
[19] A. V. Scherbakov, A. S. Salasyuk, A. V. Akimov, X. Liu, M. Bombeck, C. Brüggemann, D. R. Yakovlev, V. F. Sapega, J. K. Furdyna, and M. Bayer, Coherent Magnetization Precession in Ferromagnetic (Ga,Mn)As Induced by Picosecond Acoustic Pulses, Phys. Rev. Lett. 105, 117204 (2010).

[20] J.-W. Kim, M. Vomir, and J.-Y Bigot, Ultrafast Magnetoacoustics in Nickel Films, Phys. Rev. Lett. 109, 166601 (2012).

[21] Y. Yahagi, B. Harteneck, S. Cabrini, and H. Schmidt, Controlling nanomagnet magnetization dynamics via magnetoelastic coupling, Phys. Rev. B 90, 140405(R) (2014).

[22] J. Janušonis, C. L. Chang, P. H. M. van Loosdrecht, and R. I. Tobey, Frequency tunable surface magneto elastic waves, Appl. Phys. Lett. 106, 181601 (2015).

[23] J. V. Jäger, A. V. Scherbakov, B. A. Glavin, A. S. Salasyuk, R. P. Campion, A. W. Rushforth, D. R. Yakovlev, A. V. Akimov, and M. Bayer, Resonant driving of magnetization precession in a ferromagnetic layer by coherent monochromatic phonons, Phys. Rev. B 92, 020404(R) (2015).

[24] L. Thevenard, I. S. Camara, S. Majrab, M. Bernard, P. Rovillain, A. Lemaître, C. Gourdon, and J.-Y. Duquesne, Precessional magnetization switching by a surface acoustic wave, Phys. Rev. B 93, 134430 (2016).

[25] V. Sampath, N. D’Souza, D. Bhattacharya, G. M. Atkinson, S. Bandyopadhyay, and J. Atulasimha, Acoustic-wave-induced magnetization switching of magnetostrictive nanomagnets from single-domain to nonvolatile vortex states, Nano Lett. 16, 5681 (2016).

[26] A. Crut, P. Maioli, N. Del Fatti, and F. Vallee, Acoustic vibrations of metal nano-objects: time-domain investigations, Phys. Rep. 549, 1 (2015).

[27] T. D. Krauss and F. W. Wise, Coherent Acoustic Phonons in a Semiconductor Quantum Dot, Phys. Rev. Lett. 79, 5102 (1997).

[28] A. Devos, F. Poinsotte, J. Groenen, O. Dehaese, N. Bertru, and A. Ponchet, Strong Generation of Coherent Acoustic Phonons in Semiconductor Quantum Dots, Phys. Rev. Lett. 98, 207402 (2007).

[29] A. Bartels, T. Dekorsy, H. Kurz, and K. Köhler, Coherent Zone-Folded Longitudinal Acoustic Phonons in Semiconductor Superlattices: Excitation and Detection, Phys. Rev. Lett. 82, 1044 (1999).

[30] C.-K. Sun, J.-C. Liang, and X.-Y. Yu, Coherent Acoustic Phonon Oscillations in Semiconductor Multiple Quantum Wells with Piezoelectric Fields, Phys. Rev. Lett. 84, 179 (2000).

[31] A. S. Salasyuk, A. V. Rudkovskaya, A. P. Danilov, B. A. Glavin, S. M. Kukhtaruk, M. Wang, A. W. Rushforth, P. A. Nekludova, S. V. Sokolov, A. A. Elistratov et al., Generation of a localized microwave magnetic field by coherent phonons in a ferromagnetic nanograting, Phys. Rev. B 97, 060404(R) (2018).

[32] A. V. Akimov, C. L. Poyser and A. J. Kent, Review of microwave electro-phononics in semiconductor nanostructures, Semicond. Sci. Technol. 32, 053003 (2017).

[33] A. V. Akimov, A. V. Scherbakov, D. R. Yakovlev, and M. Bayer, Picosecond acoustics in semiconductor optoelectronic nanostructures, Ultrasonics 56, 122 (2015).

[34] H. J. Maris, Picosecond Ultrasonics, Sci. Am. 278, 86 (1998).

[35] K. H. Lin, C. M. Lai, C. C. Pan, J. I. Chyi, J. W. Shi, S. Z. Sun, C. F. Chang, and C. K. Sun, Spatial manipulation of nanoacoustic waves with nanoscale spot sizes, Nat. Nanotech. 2, 704 (2007).
[36] P.-A. Mante, Y.-R. Huang, S.-C. Yang, T.-M. Liu, A. A. Maznev, J.-K. Sheu, and C.-K. Sun, THz acoustic phonon spectroscopy and nanoscopy by using piezoelectric semiconductor heterostructures, Ultrasonics 56, 52 (2015).

[37] C.C. Shen, M. Y. Weng, J. K. Sheu, Y. T. Yao, and C. K. Sun, In situ monitoring of chemical reactions at a solid-water interface by femtosecond acoustics, J. Phys. Chem. Lett. 8, 5430 (2017).

[38] T. Dehoux, M. Abi Ghanem, O. F. Zouani, J.-M. Rampnoux, Y. Guillet, S. Dilhaire, M.-C. Durrieu, and B. Audoin, All-optical broadband ultrasonography of single cells, Sci. Rep. 5, 8650 (2015).

[39] C. Mechri, P. Ruello, J. M. Breteau, M. R. Baklanov, P. Verdonck, V. Gusev, Depth profiling of elastic inhomogeneities in transparent nanoporous low- $k$ materials by picosecond ultrasonic interferometry, Appl. Phys. Lett. 95, 091907 (2009).

[40] G. V. Hartlanda, Coherent vibrational motion in metal particles: Determination of the vibrational amplitude and excitation mechanism, J. Chem. Phys. 116, 8048 (2002).

[41] M. A. van Dijk, M. Lippitz, and M. Orrit, Detection of Acoustic Oscillations of Single Gold Nanospheres by Time-Resolved Interferometry, Phys. Rev. Lett. 95, 267406 (2005).

[42] S. O. Mariager, D. Khakhulin, H. T. Lemke, K. S. Kjær, L. Guerin, L. Nuccio, C. B. Sørensen, M. M. Nielsen, and R. Feidenhans, Direct observation of acoustic oscillations in InAs nanowires, Nano Lett. 10, 2461 (2010).

[43] A. Bruchhausen, R. Gebs, F. Hudert, D. Issenmann, G. Klatt, A. Bartels, O. Schecker, R. Waitz, A. Erbe et al., Subharmonic Resonant Optical Excitation of Confined Acoustic Modes in a Free-Standing Semiconductor Membrane at GHz Frequencies with a High-Repetition-Rate Femtosecond Laser, Phys. Rev. Lett. 106, 077401 (2011).

[44] M. Schubert, M. Grossmann, C. He, D. Brick, P. Scheel, O. Ristow, V. Gusev, and T. Dekorsy, Generation and detection of gigahertz acoustic oscillations in thin membranes, Ultrasonics 56, 109 (2015).

[45] C. He, M. Grossmann, D. Brick, M. Schubert, S. V. Novikov, C. T. Foxon, V. Gusev, A. J. Kent, and T. Dekorsy, Study of confined coherent acoustic phonon modes in a free-standing cubic GaN membrane by femtosecond spectroscopy, Appl. Phys. Lett. 107, 112105 (2015).

[46] M. F. Pascual Winter, G. Rozas, A. Fainstein, B. Jusserand, B. Perrin, A. Huynh, P. O. Vaccaro, and S. Saravanan, Selective Optical Generation of Coherent Acoustic Nanocavity Modes, Phys. Rev. Lett. 98, 265501 (2007).

[47] A.K. Geim and I.V. Grigorieva, Van der Waals heterostructures, Nature 499. 419 (2013).

[48] A. C. Ferrari, F. Bonaccorso, V. Fal'ko, K. Novoselov, S. Roche, P. Bøggild, S. Borini, F. H. L. Koppens, V. Palermo, N. Pugno et al., Science and technology roadmap for graphene, related two-dimensional crystals, and hybrid systems, Nanoscale 7, 4598 (2015).

[49] Q. H. Wang QH, K. Kalantar-Zadeh, A. Kis, J. N. Coleman, and M. S. Strano, Electronics and optoelectronics of twodimensional transition metal dichalcogenides, Nat Nanotechnol. 7, 699 (2012).

[50] S. Z. Butler, S. M. Hollen, L. Cao, Y. Cui, J. A. Gupta, H. R. Gutiérrez, T. F. Heinz, S. S. Hong, J. Huang, A. F. Ismach et al., Progress, challenges, and opportunities in twodimensional materials beyond graphene, ACS Nano 7, 2898 (2013). 
[51] N. T. Hung, A. R. T. Nugraha, and R. Saito, Two-dimensional InSe as a potential thermoelectric material, Appl. Phys. Lett. 111, 092107 (2017).

[52] A. D. O'Connell, M. Hofheinz1, M. Ansmann, R. C. Bialczak, M. Lenander, E. Lucero, M. Neeley, D. Sank, H. Wang, M. Weides et al., Quantum ground state and single-phonon control of a mechanical resonator, Nature 464, 697 (2010).

[53] M. R. Armstrong, E. J. Reed, K.-Y. Kim, J. H. Glownia, W. M. Howard, E. L. Piner, and J. C. Roberts, Observation of terahertz radiation coherently generated by acoustic waves, Nat. Phys. 5, 285 (2009).

[54] S. L. Heywood, B. A. Glavin, R. P. Beardsley, A. V. Akimov, M. W. Carr, J. Norman, P. C. Norton, B. Prime, N. Priestley, and A. J. Kent, Heterodyne mixing of millimetre electromagnetic waves and sub-THz sound in a semiconductor device, Sci. Rep. 6, 30396 (2016).

[55] T. Y. Jeong, B. M. Jin, S. H. Rhim, L. Debbichi, J. Park, Y. D. Jang, H. R. Lee, D.-H. Chae, D. Lee, Y.-H. Kim et al., Coherent Lattice Vibrations in Mono- and Few-Layer WSe2, ACS Nano 10, 5560 (2016).

[56] B. He, C. Zhang, W. Zhu, Y. Li, S. Liu, X. Zhu, X. Wu, X. Wang, H. Wen, and M. Xiao, Coherent optical phonon oscillation and possible electronic softening in WTe2 crystals, Sci. Rep. 6, 30487 (2016)

[57] S. Ge, X. Liu, X. Qiao, Q. Wang, Z. Xu, J. Qiu, P.-H. Tan, J.Zhao, and D. Sun, Coherent longitudinal acoustic phonon approaching $\mathrm{THz}$ frequency in multilayer molybdenum disulphide, Sci. Rep. 4, 5722 (2014).

[58] R. Beardsley, A. V. Akimov, S. Sandeep, Z. R. Kudrynskyi, Z. D. Kovalyuk, A. Patanè, and A. J. Kent, Nanomechanical probing of the layer/substrate interface of an exfoliated InSe sheet on sapphire, Sci. Rep. 6, 26970 (2016).

[59] G. W. Mudd, S. A. Svatek, T. Ren, A. Patanè, O. Makarovsky, L. Eaves, P. H. Beton, Z. D. Kovalyuk, G. V. Lashkarev, Z. R. Kudrynskyi et al., Tuning the bandgap of exfoliated InSe nanosheets by quantum confinement, Adv. Mater. 25, 5714 (2013).

[60] D. A. Bandurin, A. V. Tyurnina, G. L. Yu, A. Mishchenko, V. Zólyomi, S. V. Morozov, R. K. Kumar, R. V. Gorbachev, Z. R. Kudrynskyi, S. Pezzini et al., High electron mobility, quantum Hall effect and anomalous optical response in atomically thin InSe, Nat. Nanotechnol. 12, 223 (2017).

[61] J. F. Sánchez-Royo, G. Muñoz-Matutano, M. Brotons-Gisbert, J. P. Martínez-Pastor, A. Segura, A. Cantarero, R. Mata, J. Canet-Ferrer, G. Tobias, E. Canadell et al., Electronic structure, optical properties, and lattice dynamics in atomically thin indium selenide flakes, Nano Research 7, 1556 (2014).

[62] T. Pandey, D. S. Parker and L. Lindsay, Ab initio phonon thermal transport in monolayer InSe, GaSe, GaS, and alloys, Nanotechnology 28, 455706 (2017).

[63] A. S. Nissimagoudar, J. Ma, Y. Chen, and W. Li, Thermal transport in monolayer InSe, J. Phys.: Condens. Matter 29, 335702 (2017).

[64] M. Gatulle, M. Fischer, and A. Chevy, Elastic constants of the layered compounds $\mathrm{GaS}, \mathrm{GaSe}$, InSe, and their pressure dependence, I Experimental part, Phys. Status Solidi B 119, 327 (1983).

[65] S. L. Rumyantsev, M. E. Levinshtein, A. D. Jackson, S. N. Mohammad, G. L. Harris, M. G. Spencer, and M. S. Shur, in Properties of Advanced Semiconductor Materials GaN, AlN,
InN, BN, SiC, SiGe, edited by M. E. Levinshtein, S. L. Rumyantsev, and M. S. Shur (Wiley, New York, 2001), pp. 67-92.

[66] F. J. Manjon, Y. van der Vijver, A. Segura and V. Munoz, Pressure dependence of the refractive index in InSe, Semicond. Sci. Technol. 15, 806 (2000).

[67] C. Thomsen, H. T. Grahn, H. J. Maris, and J. Tauc, Surface generation and detection of phonons by picosecond light pulses, Phys. Rev. B 34, 4129 (1986).

[68] O. B. Wright, Ultrafast nonequilibrium stress generation in gold and silver, Phys. Rev. B 49, 9985 (1994).

[69] E. S. K. Young, A. V. Akimov, R. P. Campion, A. J. Kent, and V. Gusev, Picosecond strain pulses generated by a supersonically expanding electron-hole plasma in GaAs, Phys. Rev. B 86, 155207 (2012).

[70] A. Bartels, R. Cerna, C. Kistner, A. Thoma, F. Hudert, C. Janke, and T. Dekorsy, Ultrafast time-domain spectroscopy based on high-speed asynchronous optical sampling, Rev. Sci. Instrum. 78, 035107 (2007).

[71] N. Balakrishnan, Z. R. Kudrynskyi, E. F. Smith, M. W. Fay, O. Makarovsky, Z. D. Kovalyuk, L. Eaves, P. H. Beton, and A. Patanè, Engineering p-n junctions and bandgap tuning of InSe nanolayers by controlled oxidation, 2D Mater. 4, 025043 (2017).

[72] O. Matsuda, O. B. Wright, D. H. Hurley, V. Gusev, and K. Shimizu, Coherent shear phonon generation and detection with picosecond laser acoustics, Phys. Rev. B 77, 224110 (2008).

[73] B. A. Auld, Acoustic Waves and Fields in Solids, 2nd ed. (Krieger, Malalabar, 1990), Vol. I.

[74] G. Tas, J. J. Loomis, H. J. Maris, A. A. Bailes, and L. E. Seiberling, Picosecond ultrasonics study of the modification of interfacial bonding by ion implantation, Appl. Phys. Lett. 72, 2235 (1998).

[75] J. Serrano, A. Bosak, R. Arenal, M. Krisch, K. Watanabe, T. Taniguchi, H. Kanda, A. Rubio, and L. Wirtz, Vibrational Properties of Hexagonal Boron Nitride: Inelastic X-Ray Scattering and Ab Initio Calculations, Phys. Rev. Lett. 98, 095503 (2007).

[76] V. Panella, G. Carlotti, G. Socino, L. Giovannini, M. Eddrief, K. Amimer, and C. Sebenne, Brillouin scattering study of epitaxial InSe films grown on the surface, J. Phys.: Condens. Matter 9, 5575 (1997).

[77] S. J. Magorrian, V.Z'olyomi and V. I. Fal'ko, Electronic and optical properties of two-dimensional InSe from a DFT-parametrized tight-binding model, Phys. Rev. B 94, 245431 (2016).

[78] Y. Zhirko, V. Trachevsky, and Z. Kovalyuk, On the Possibility of Layered Crystals Application for Solid State Hydrogen Storages - InSe and GaSe Crystals, Hydrogen Storage, edited by J. Liu, (InTech, Croatia, 2012).

[79] T. Björkman, A. Gulans, A. V. Krasheninnikov, and R. M. Nieminen, van der Waals Bonding in Layered Compounds from Advanced Density-Functional First-Principles Calculations, Phys. Rev. Lett. 108, 235502 (2012).

[80] T. Ayadi, L. Debbichi, M. Said, and S. Lebègue, An ab initio study of the electronic structure of indium and gallium chalcogenide bilayers, J. Chem. Phys. 147, 114701 (2017).

[81] R. Schwarcz, M. A. Kanehisa, M. Jouanne, J. F. Morhange, and M. Eddrief, Evolution of Raman spectra as a function of layer thickness in ultra-thin InSe films, J. Phys.: Condens. Matter 14, 967 (2002).

[82] V. V Korolkov, S. A Svatek, A. Summerfield, J. Kerfoot, L. Yang, T. Taniguchi, K. Watanabe, N. R. Champness, N. A. Besley, and P. H. Beton, van der Waals-induced chromatic shifts in 
hydrogen-bonded two-dimensional porphyrin arrays on boron nitride, ACS Nano 9, 10347 (2015).

[83] V. V Korolkov, M. Baldoni, K. Watanabe, T. Taniguchi, E. Besley, and P. H. Beton, Supramolecular heterostructures formed by sequential epitaxial deposition of two-dimensional hydrogen-bonded arrays, Nat. Chem. 9, 1191 (2017).

[84] J. Cuffe, O. Ristow, E. Chávez, A. Shchepetov, P-O. Chapuis, F. Alzina, M. Hettich, M. Prunnila, J. Ahopelto, T. Dekorsy, and C. M. Sotomayor Torres, Lifetimes of Confined Acoustic Phonons in Ultrathin Silicon Membranes, Phys. Rev. Lett. 110, 095503 (2013).

[85] A. G. Voronovich, Small-slope approximation in wave scattering by rough surfaces, Sov. Phys. JETP. 62, 65 (1985).

[86] S. L. Broschat and E. I. Thorsos, An investigation of the small slope approximation for scattering from rough surfaces. Part II. Numerical studies, J. Acoust. Soc. Am. 101, 2615 (1997).
[87] Y.-C. Wen, C.-L. Hsieh, K.-H. Lin, H.-P. Chen, S.-C. Chin, C.-L. Hsiao, Y.-T. Lin, C.-S. Chang, Y.-C. Chang, L.-W. Tu, and C.-K. Sun, Specular Scattering Probability of Acoustic Phonons in Atomically Flat Interfaces, Phys. Rev. Lett. 103, 264301 (2009).

[88] T. Hu, J. Zhou and J. Dong, Strain induced new phase and indirect-direct band gap transition of monolayer InSe, Phys. Chem. Chem. Phys. 19, 21722 (2017).

[89] M. Grossmann, M. Schubert, C. He, D. Brick, E. Scheer, M. Hettich, V. Gusev and T. Dekorsy, Characterization of thin-film adhesion and phonon lifetimes in $\mathrm{Al} / \mathrm{Si}$ membranes by picosecond ultrasonics, New J. Phys. 19, 053019 (2017).

[90] J.-M. Baik, and R. B. Thompson, Ultrasonic Scattering from Imperfect Interfaces: A Quasi-Static Model, J. Nondestructive Evaluation 4, 177 (1984). 